\title{
A variant of the Hough Transform for the combined detection of corners, segments, and polylines
}

\author{
Pilar Bachiller-Burgos ${ }^{*}$ (D), Luis J. Manso and Pablo Bustos
}

\begin{abstract}
The Hough Transform (HT) is an effective and popular technique for detecting image features such as lines and curves. From its standard form, numerous variants have emerged with the objective, in many cases, of extending the kind of image features that could be detected. Particularly, corner and line segment detection using HT has been separately addressed by several approaches. To deal with the combined detection of both image features (corners and segments), this paper presents a new variant of the Hough Transform. The proposed method provides an accurate detection of segment endpoints, even if they do not correspond to intersection points between line segments. Segments are detected from their endpoints, producing not only a set of isolated segments but also a collection of polylines. This provides a direct representation of the polygonal contours of the image despite imperfections in the input data such as missing or noisy feature points. It is also shown how this proposal can be extended to detect predefined polygonal shapes. The paper describes in detail every stage of the proposed method and includes experimental results obtained from real images showing the benefits of the proposal in comparison with other approaches.
\end{abstract}

Keywords: Hough Transform, Corner detection, Line segment detection, Polyline detection

\section{Introduction}

Corner and line segment detection is essential for many computer vision applications. Corner detection is used in a wide variety of applications such as camera calibration [1], target tracking [2], image stitching [3], and 3D modeling [4]. The detection of line segments can also be helpful in several problems including robot navigation [5], stereo analysis [6], and image compression [7]. Going a step further, the combined detection of both image features may result in the identification of polygonal structures, which plays an important role in many applications such as aerial imagery analysis $[8,9]$ and cryo-electron microscopy [10].

The Hough Transform (HT) [11] is one of the most popular and widely used techniques for detecting image features such as lines, circles, and ellipses [12]. Its effectiveness emerges from its tolerance to gaps in feature boundaries and its robustness against image noise. These

*Correspondence: pilarb@unex.es

Department of Computer and Communication Technology, University of

Extremadura, Avda. de la Universidad s.n., 10003 Caceres, Spain properties make HT a powerful tool for detecting image features in real images. To exploit its benefits, we propose a new variant of the HT, called HT3D, for the combined detection of corners and line segments.

The basic form of the Hough Transform, also known as the Standard Hough Transform (SHT) [13], has been established as the main technique for straight line detection [14-16]. The line detection problem is transformed by the SHT into a peak detection problem. Each feature point, obtained from a previous pre-processing stage such as edge detection, is mapped to a sine curve following the expression $d=x \cos (\theta)+y \sin (\theta)$. The parameter space defined by $(\theta, d)$ is discretized into finite intervals, called accumulator cells or bins, giving rise to the Hough space (also referred to as the accumulator array). Using this discrete version of the parameter space, each feature point votes for the lines passing through it by incrementing the accumulator cells that lie along the corresponding curve. After this voting process, lines are located at those positions forming local maxima in the Hough space. 
The SHT for straight line detection does not provide a direct representation of line segments, since feature points are mapped to infinite lines in the parameter space. To deal with segment representation, we propose a 3D Hough space that, unlike SHT, uses several bins to represent each line. This 3D Hough space not only provides a segment representation but also encloses canonical configurations of two kinds of distinctive points: corners and nonintersection endpoints. Corners are points at which two line segments meet at a particular angle. Non-intersection endpoints are extreme points of line segments that do not intersect any other segment. Both types of points identify line segment boundaries, but corners can additionally be considered connection points among segments. This makes it possible to extend line segment detection to the identification of chains of segments. Thus, the proposed parameter space constitutes a suitable structure for detecting three kinds of image features: corners, line segments, and polylines.

The use of HT for individual detection of corners and line segments has been explored from different approaches over the last few decades.

For segment detection, two main approaches have been proposed. The first group of methods is based on image space verification from the information of the HT peaks. Song and Lyu [17] propose an HT-based segment detection method which utilizes both the parameter space and the image space. Using a boundary recorder for each parameter cell, the authors develop an image-based line verification algorithm. The boundary recorder of each cell includes the upper and lower boundaries which enclose all feature points contributing to that cell. Gerig [18] suggests a backmapping which links the image space and the parameter space. After the accumulation phase, the transform is used a second time to compute the location of the cell most likely to be related to each image point. This backmapping process provides a connection between edge points and Hough cells in the detection of line segments. Matas et al. [19] present PPHT (Progressive Probabilistic Hough Transform), a Monte Carlo variant of the Hough Transform. PPHT obtains line segments by looking along a corridor in the image specified by the peak in the accumulator modified during pixel voting. Nguyen et al. [20] propose a similar strategy, but in this approach, the method is based on SHT with some extensions.

The other group of methods for segment detection detects properties of segments by analyzing the data in the parameter space. Cha et al. [21] propose an extended Hough Transform, where the Hough space is formed by $2 \mathrm{D}$ planes that collect the evidence of the line segment that passes through a specific column of the input image. Using this scheme, a feature extraction method is proposed for detecting the length of a line segment. In the study by $\mathrm{Du}$ et al. [22], the authors propose a segment detection method based on the definition and analysis of the neighborhoods of straight line segments in the parameter space. In another study [23], the parameters of a line segment are obtained by analyzing the distinct distribution around a peak in the accumulator array. This distribution is called butterfly distribution due to its particular appearance. Xu and Shin [24] propose an improvement of the peak detection by considering voting values as probabilistic distributions and using entropies to estimate the peak parameters. Endpoint coordinates of a line segment are then computed by fitting a sine curve around the peak.

In relation to corner detection using $\mathrm{HT}$, various methods can be found in the literature. Davies [25] uses the Generalized Hough Transform [26] to transform each edge pixel into a line segment. Corners are found at peaks in the Hough space, where lines intersect. Barret and Petersen [27] propose a method to identify line intersection points by finding collections of peaks in the Hough space through which a given sinusoid passes. Shen and Wang [28] present a corner identification method based on the detection of straight lines passing through the origin in a local coordinate system. For detecting such lines, the authors propose a 1D Hough Transform.

Unlike other methods, our proposal provides an integrated detection of corners and line segments, which has important benefits in terms of accuracy and applicability of the results. Thus, segments are detected from previously extracted endpoints, which guarantees a better correspondence between detected and actual segments than other approaches. Corners are detected by searching for points intersecting two line segments at a given range of angles. This makes it possible to detect features that other methods miss, such as obtuse-angle corners. Besides corners, our technique detects the location of any segment endpoint with high accuracy, even if such an endpoint does not belong to any other segment. In addition, the proposed method produces chains of segments as a result of segment detection, providing a complete representation of the polygonal contours of the image despite imperfections in the input data such as missing or noisy feature points.

The rest of the paper is organized as follows. Section 2 details the proposal. Specifically, the proposed 3D Hough space and the voting process are described in Subsection 2.1. Subsection 2.2 presents the corner and endpoint detection method. In Subsection 2.3, the algorithm for segment detection and polygonal approximation is detailed. All these subsections show how to keep the computational complexity under certain limits. In addition, an analysis of the computational cost of each phase of the proposed method is included in Subsection 2.4. Subsection 2.5 describes how our proposal can be extended to detect not only arbitrary polygonal shapes but also predefined ones. Section 3 presents experimental results 
with real images, comparing the methods proposed in this paper with other approaches. Finally, Section 4 summarizes the main conclusions of this paper.

\section{The proposed method}

\subsection{The 3D Hough space}

To deal with the detection of line segments using HT, a parametrization of the geometric model of this kind of image features must be established. Thus, assume that two points $e_{i}=\left(x_{i}, y_{i}\right)$ and $e_{j}=\left(x_{j}, y_{j}\right)$ define an image segment of the line $l(d, \theta) \equiv d=x \cos (\theta)+y \sin (\theta)$. Let $p_{i}$ and $p_{j}$ be the positions of $e_{i}$ and $e_{j}$ relative to the line $l(d, \theta)$. To compute these relative positions, consider a coordinate system local to the line where the vertical axis coincides with the line and the horizontal one passes through the image origin (see Fig. 1). Using this local system, the relative position $(p)$ of a point $e=(x, y)$ of the line $l(d, \theta)$ can be computed by determining the $y$-coordinate of the point as follows:

$$
p=-x \sin (\theta)+y \cos (\theta)
$$

According to this and taking $p_{i}<p_{j}$, a point $e=(x, y)$ belongs to the segment $\overline{e_{i} e_{j}}$ if it fulfills the following two expressions:

$$
\begin{aligned}
d & =x \cos (\theta)+y \sin (\theta) \\
p_{j}> & =-x \sin (\theta)+y \cos (\theta)>=p_{i}
\end{aligned}
$$

Equation 2 determines whether the point $e$ belongs to the line $l(d, \theta)$. The additional condition expressed by Eq. 3 forces the point $e$ to be situated between $e_{i}$ and $e_{j}$.

Considering the conditions imposed by these two expressions, a line segment can be described by four parameters: $d, \theta, p_{i}$, and $p_{j}$. It is assumed that the origin of the image coordinate system is located at its center. Thus,

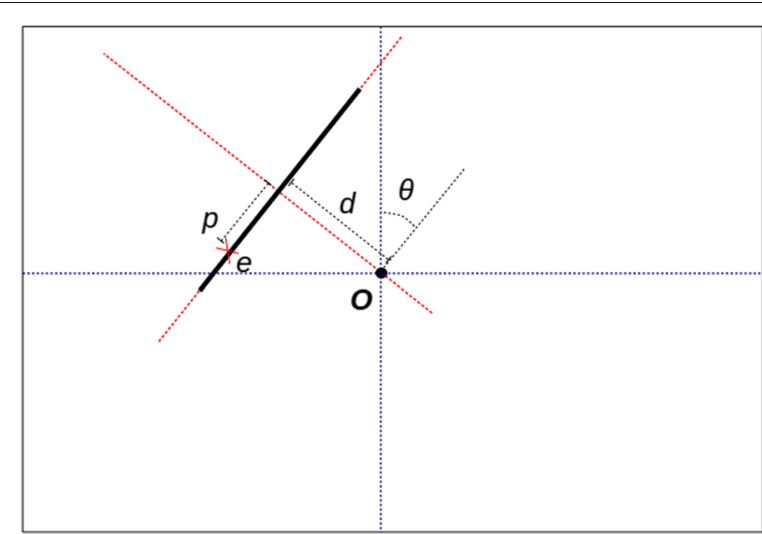

Fig. 1 Relative position $p$ of an image point $e$ to the line $/(d, \theta)$. The relative position $p$ is computed by determining the $y$-coordinate of the point $e$ in a coordinate system local to the line / (dotted red lines). It is considered that the image reference system (dotted blue lines) is situated at the image center $(O)$ $\theta \in[0, \pi)$, and $d, p_{i}, p_{j} \in[-R,+R]$, with $R$ being the half of the length of the image diagonal. This leads to a $4 \mathrm{D}$ parameter space, where each feature point $(x, y)$ in the image contributes to those points $\left(\theta, d, p_{i}, p_{j}\right)$ of the parameter space that verify Eqs. 2 and 3.

This parameter space provides a complete representation of image line segments. Nevertheless, in practice, the use of a 4D structure could make the detection method excessively demanding in terms of memory and time. To tackle this problem, a 3D parameter space representing a subset of line segments is proposed (Fig. 2). This segment subset is defined by the point pairs $e_{0}-e_{j}$, with $e_{0}$ being a fixed endpoint situated at the smallest line position $\left(p_{0}=-R\right)$ and $e_{j}$ a variable endpoint situated at any position $p$ within a line. Thus, the proposed Hough space is parametrized by $(\theta, d, p)$, with $\theta$ and $d$ being the parameters of the line representation $(l(d, \theta) \equiv d=x \cos (\theta)+$ $y \sin (\theta))$ as in the standard HT. The additional parameter $p$ defines the relative position of $e_{j}$ for a given segment $\overline{e_{0} e_{j}}$ of the line $l(d, \theta)$. Using this representation, any point $(x, y)$ contributes to those points $(\theta, d, p)$ in the Hough space verifying Eq. 2 and the expression of Eq. 3, taking $p_{i}$ as $-R$ and $p_{j}$ as $p$. Since the parameter $p$ ranges from $-R$ to $R$, the last inequation of expression 3 is always true. Thus, such an expression can be rewritten as follows:

$$
p>=-x \sin (\theta)+y \cos (\theta)
$$

Although this 3D Hough space does not directly represent every possible segment of the image, it allows computing the total number of points included in any given segment (see Fig. 2). For instance, take a segment of a given line $l\left(d_{l}, \theta_{l}\right)$ defined by the endpoints $e_{i}=\left(x_{i}, y_{i}\right)$ and $e_{j}=\left(x_{j}, y_{j}\right)$. Assuming that, for the line $l, p_{i}$ is lower than $p_{j}$, it can be stated that segment $\overline{e_{0} e_{i}}$ is included in $\overline{e_{0} e_{j}}$. Thus, the number of points belonging to $\overline{e_{i} e_{j}}$, which is denoted by $H_{i \leftrightarrow j}$, can be computed as:

$$
H_{i \leftrightarrow j}=\left|H\left(\theta_{l}, d_{l}, p_{i}\right)-H\left(\theta_{l}, d_{l}, p_{j}\right)\right|
$$

with $H$ being the proposed 3D Hough space.

This last equation leads into the quantification of the segment strength, which for two points $e_{i}$ and $e_{j}$ can be expressed as:

$$
s s\left(e_{i}, e_{j}\right)=H_{i \leftrightarrow j} / \sqrt{\left(x_{i}-x_{j}\right)^{2}+\left(y_{i}-y_{j}\right)^{2}}
$$

The values of $s s\left(e_{i}, e_{j}\right)$ range from 0 to 1 . The higher the value, the higher the likelihood of the existence of a line segment between $e_{i}$ and $e_{j}$.

\subsubsection{The voting process}

In order to form the discrete 3D Hough space $H$, the parameters $\theta, d$, and $p$ are discretized assuming resolutions of $\Delta \theta$ for $\theta, \Delta d$ for $d$, and $\Delta p$ for $p$. To carry out the voting process, for every orientation plane $(H(\theta))$, each point must vote for those cells that verify Eqs. 2 

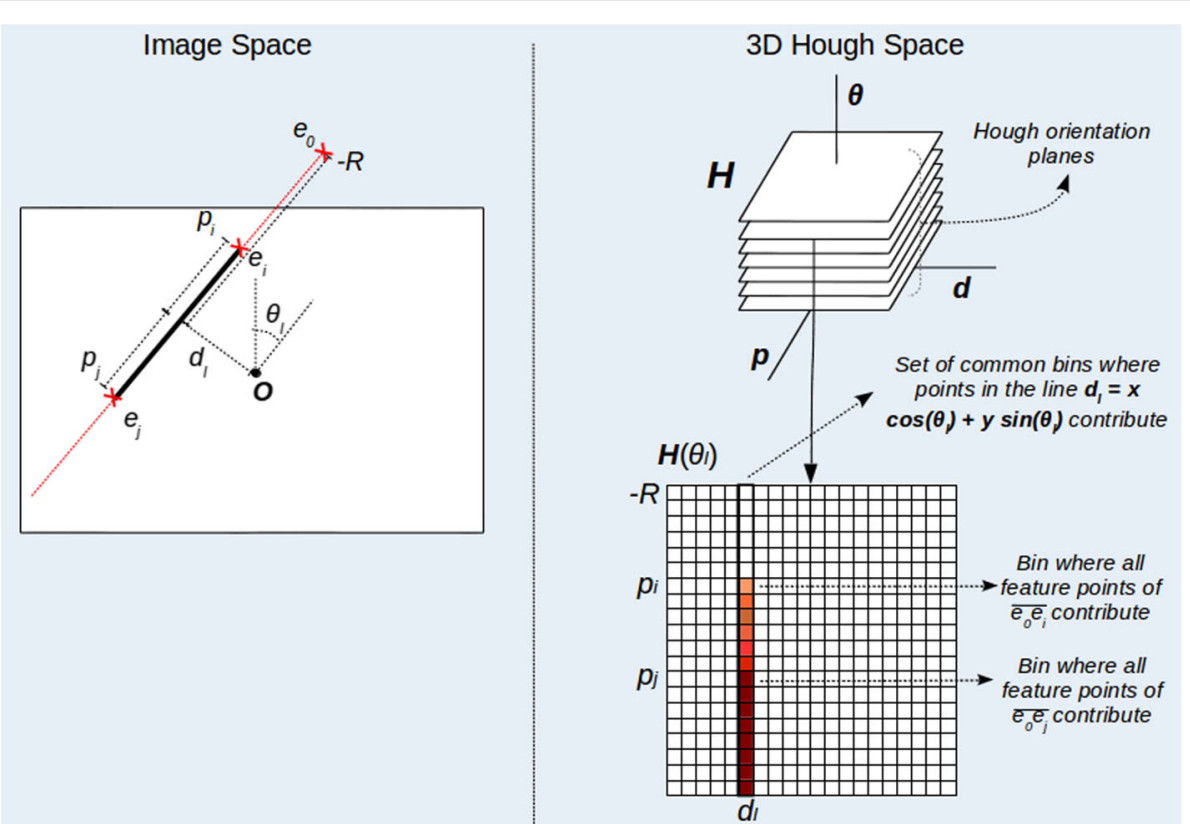

Fig. 2 3D Hough space and line segment representation. Points of a line $/\left(d_{l}, \theta_{l}\right)$ contribute to a subset of cells situated at $d_{l}$ in the Hough plane $H\left(\theta_{l}\right)$. Specifically, a point $e_{i}$ situated at a position $p_{i}$ relative to the line $l\left(d_{l}, \theta_{l}\right)$ contributes to the subset of cells for which $p>=p_{i}$. As a consequence, the bin $H\left(\theta_{l}, d_{l}, p_{i}\right)$ contains the total number of feature points of the segment $\overline{e_{0} e_{i}}$. Likewise, the bin $H\left(\theta_{l}, d_{1}, p_{j}\right)$ contains the number of feature points of $\overline{e_{0} e_{j}}$. Thus, the difference between the contents of both bins provides the total number of feature points of the segment $\overline{e_{i} e_{j}}$

and 4. This process is computationally expensive, since it entails running two nested loops for every feature point. However, this complexity can be reduced by dividing the voting process into two stages: (a) points only vote for the first segment they could belong to in every orientation plane, i.e., $p$ is computed using only the equality of expression 4; (b) starting from the second discrete value of $p\left(p_{d}=p_{\text {min }}+1\right)$, each cell in $H\left(\theta_{d}, d_{d}, p_{d}\right)$ accumulates with $H\left(\theta_{d}, d_{d}, p_{d}-1\right)$ for $\theta_{d} \in\left[\theta_{\min }, \theta_{\text {max }}\right]$ and $d_{d} \in\left[d_{\min }, d_{\max }\right]$. Algorithms 1 and 2 describe these two stages. As it can be observed, in the second stage, each cell in $H$ is saturated to $\Delta p$ before accumulation takes place. Cell saturation means that every cell crossed by a segment must contain a minimum contribution for it to be considered an actual segment. This reduces false positives in the detection of segments caused by the contribution of different line points to common bins.

To improve the efficiency of this voting scheme, additional optimizations can be introduced. Thus, as suggested in some approaches, the local orientation of the edges can be used to limit the range of angles over which a point should vote [26]. This reduces the computational cost of Algorithm 1. In relation to Algorithm 2, a possible optimization consists of storing, for every line, the minimum value of $p_{d}$ for which points have voted and using this value to start each accumulation loop. This means that if a line of the Hough space has no points, it will not be taken into account at the accumulation stage, and so the time needed to execute that stage is reduced.
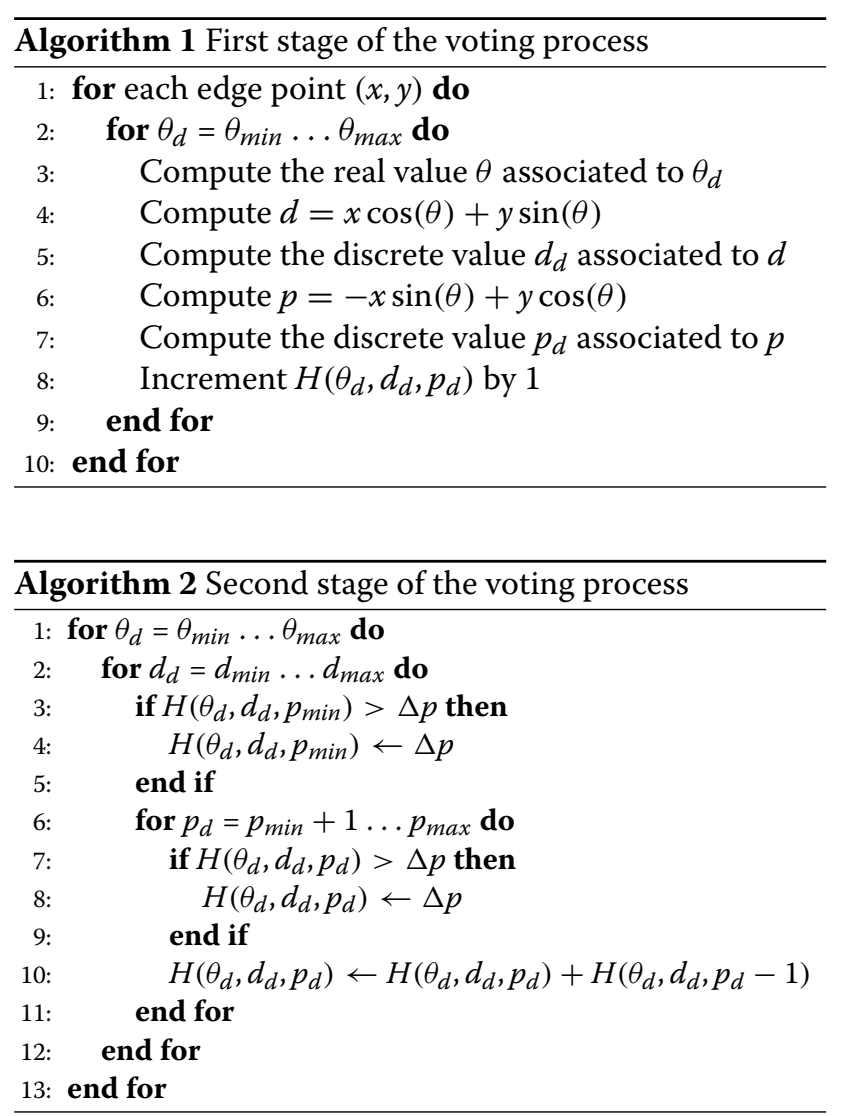


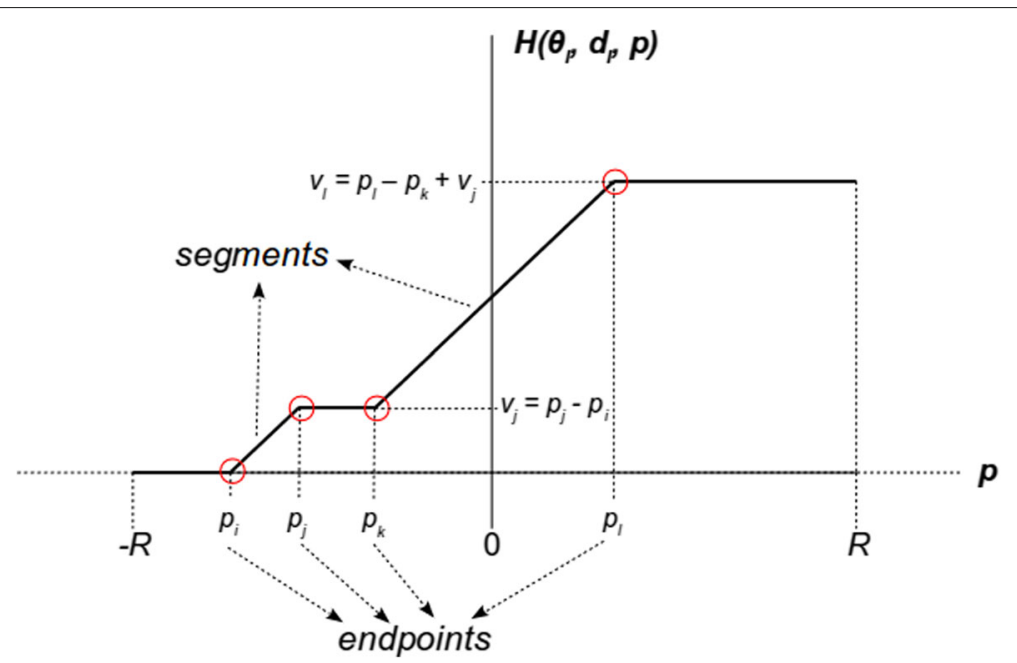

Fig. 3 Representation of two segments of a line $/\left(d_{l}, \theta_{l}\right)$ in the 3D Hough space

\subsubsection{Interpreting the 3D Hough space}

Using the proposed parameter space, segment detection cannot be treated as a traditional peak detection problem, since each cell only contains information about one of the two endpoints of a given segment. Complete segment parametrization is held in pairs of cells with common values of $\theta$ and $d$. Thus, segment detection is accomplished by searching for pairs of cells belonging to the same line that exhibit a ratio of close to 1 between the difference of content (number of points included between segment endpoints) and the difference of position (segment length). To avoid detecting segment parts as independent segments, it is required that a line segment may not belong to a longest segment. Figure 3 shows this idea. This figure graphically depicts the content of the cells associated with a given line $l\left(d_{l}, \theta_{l}\right)$ using a continuous function in parameter space for simplification. The evolution of cell contents shows the existence of two (ideal) line segments: the first one situated between line positions $p_{i}$ and $p_{j}$ and the other one between $p_{k}$ and $p_{l}$.

This strategy for segment detection presents two main drawbacks. Firstly, the computational cost of checking the segment feature for every pair of cells of each line is excessive. In addition, in the discrete parameter space, the accuracy of segment representation could not be enough to obtain useful results. To solve both problems, we propose detecting segment endpoints instead of complete segments and using the resulting positions to confirm the existence of line segments. The benefit of applying this process is twofold: in the first place, segment endpoints can be detected from local cell patterns, reducing considerably the cost of feature detection; secondly, starting from the initial positions of points detected in the parameter space, segment endpoints can be accurately located in the image space computing the pixel locations that maximize an endpoint measure in a local environment.

The following subsections describe this approach to feature detection. Starting from segment endpoints detection (Subsection 2.2), it is detailed how to extract line segments from the 3D Hough space with good precision (Subsection 2.3) as well as more complex image features (Subsection 2.5).

\subsection{Detection of segment endpoints}

In our proposal, segment endpoints are classified into two types of distinctive points: corners and non-intersection endpoints. A corner point is a point that belongs to at least two segments. A non-intersection endpoint is an extreme point of a segment which does not belong to any other segment. Figure 4 shows some representative examples of both kinds of points.

The detection process of corners and non-intersection endpoints can be visualized by focusing on the accumulator subset corresponding to the particular orientation associated to an image line segment. Assuming that in the resulting 2D array the parameter $d$ corresponds to the horizontal dimension and $p$ to the vertical one, an image line segment only contributes to a vertical segment of cells in the corresponding orientation plane of the Hough space, since the value of the parameter $d$ remains constant for all the points on the line. This phenomenon produces the canonical configuration of cells in Figs. $5 b$ and $6 b$ for pieces of image segments containing a non-intersection endpoint (Fig. 5a) or a corner (Fig. 6a). In these configurations, black cells represent cells whose values differ from the ones above them, white cells are cells in the opposite situation and gray cells correspond to cells whose content is not significant for corner or endpoint detection. As it can be observed, except the one associated 


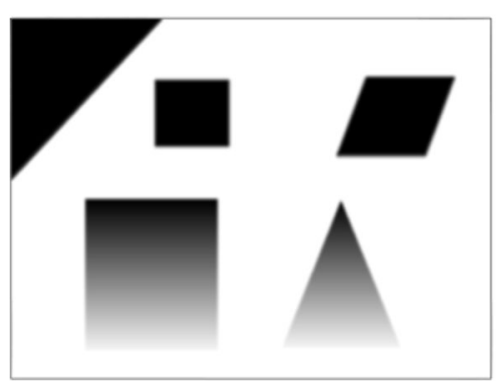

a

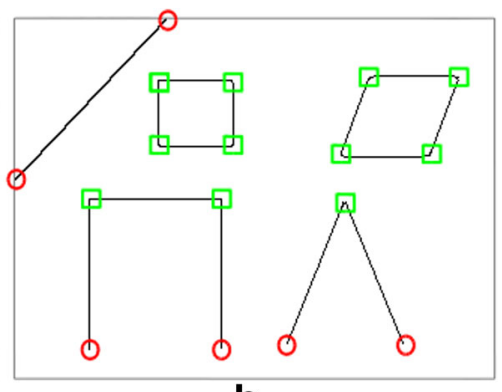

b

Fig. 4 Examples of the different kinds of line segment endpoints considered in the proposed detection method. a Original gray image. b Line segments obtained by an edge detection process. In this second image, corners are marked with green squares and non-intersection endpoints with red circles. Corners correspond to the crossing points of two line segments at a certain range of angles. Non-intersection endpoints appear in the intersections of line segments with the image boundaries as well as in the start and end points of the open polylines resulting from image areas with progressive gray-level variations

with a corner, black cells must be situated beside white cells to reduce the number of false corners and endpoints caused by nearby image segments. In addition, since the proposed parameter space only covers the orientation range $[0, \pi)$, it is also considered a flipped version of each Hough segment representation.

According to the correspondence between a line segment in the image and Hough spaces, the detection of corners and non-intersection endpoints can be solved by searching for the aforementioned configurations of segments in the 3D Hough space. To check these configurations, cells are grouped into vertical full (black cells) or empty (white cells) segments. A vertical full segment verifies that the difference between its last and first cell is greater than a threshold $\tau_{F}$. On the other hand, this

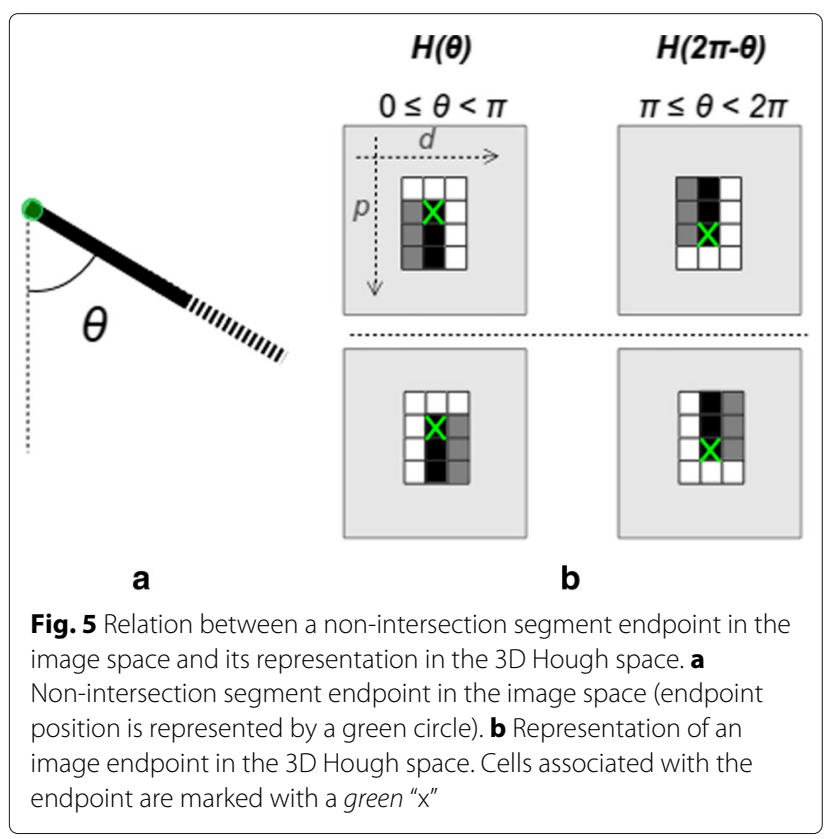

difference must be lower than a near to zero threshold $\left(\tau_{E}\right)$ in an empty segment. Thus, given a certain position in the Hough space $\left(\theta_{d}, d_{d}, p_{d}\right)$ and taking $\eta$ as the number of cells of a full piece of segment in the Hough representation, to verify if that position contains, for instance, a corner, the following must be fulfilled:

$$
H\left(\theta_{d}, d_{d}, p_{d}+\eta-1\right)-H\left(\theta_{d}, d_{d}, p_{d}-1\right)>\tau_{F}
$$

and

$$
H\left(\theta_{d}, d_{d}+1, p_{d}+\eta-1\right)-H\left(\theta_{d}, d_{d}+1, p_{d}\right)<\tau_{E}
$$

or

$$
H\left(\theta_{d}, d_{d}-1, p_{d}+\eta-1\right)-H\left(\theta_{d}, d_{d}-1, p_{d}\right)<\tau_{E}
$$

for the plane $H\left(\theta_{d}\right)$ and the same expressions for the corresponding position of the plane $H\left((\theta+\varphi)_{d}\right)$, given a certain range of $\varphi$ and assuming $\theta<\pi$ and $(\theta+\varphi)<\pi$.

The value of $\eta$ should be chosen according to the need for detecting approximations of line segment endpoints in curvilinear shapes. Small values of $\eta$ provide additional feature points that are the result of changes of curvature on curvilinear boundaries. Nevertheless, these points are less stable than rectilinear corners and segment endpoints. Thus, if the stability of detected features along image sequences is essential, greater values of $\eta$ must be chosen.

\subsubsection{Reducing the computational complexity}

The verification of the segment configurations of corners and endpoints over the whole 3D Hough space entails many computations due to its size. Nevertheless, there are two issues that should be taken into account in the implementation of this detection process. The first one is that if a line has few points, it is not necessary to search for corners and endpoints over its cells, since it will contain no segment. This situation can be verified checking the cell $H\left(\theta_{d}, d_{d}, p_{\max }\right)$ for any $\theta_{d}$ and $d_{d}$ defining a line, since such 


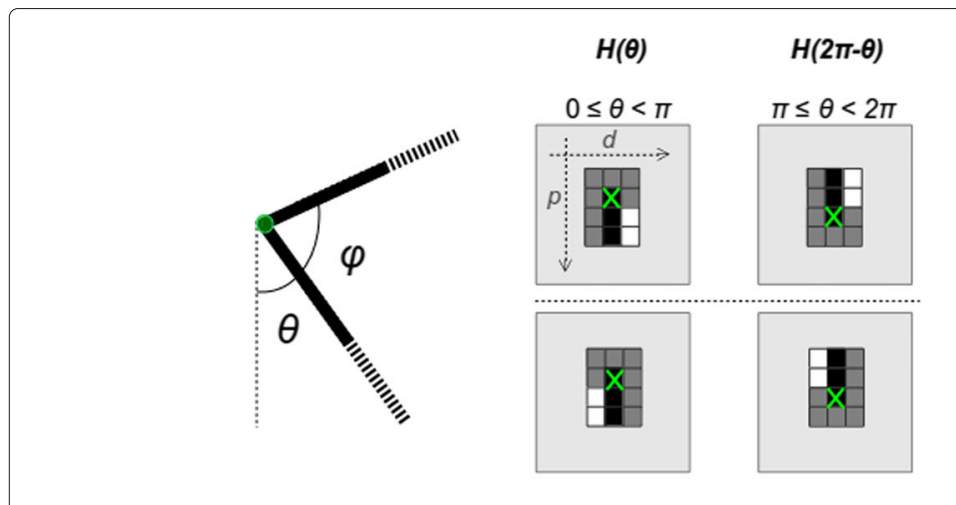

a

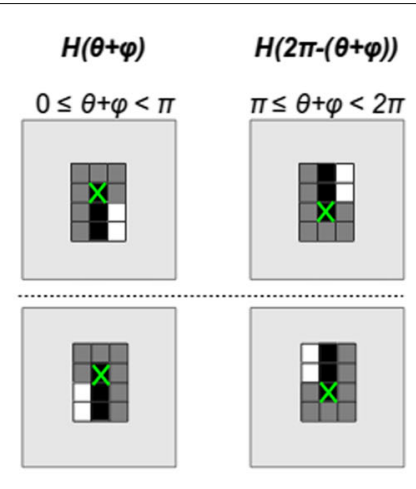

b

Fig. 6 Relation between an image corner and its representation in the 3D Hough space. a Image corner (corner position is represented by a green circle). b Representation of an image corner in the 3D Hough space. Cells associated with the corner are marked with a green " $x$ "

a cell contains the total number of points belonging to that line. Another important question that should be considered is that, in the discrete parameter space, the relative positions of the cells crossed by a piece of image segment remain the same in several consecutive orientation planes. Thus, it is not necessary to run the detection process for every orientation plane, but only for those planes representing a certain increase in angle $(\phi)$.

To compute $\phi$, consider the positions $\left(d_{1}, p_{1}\right)$ and $\left(d_{2}, p_{2}\right)$ (Eqs. 1 and 2) in a given orientation plane $H(\theta)$ of

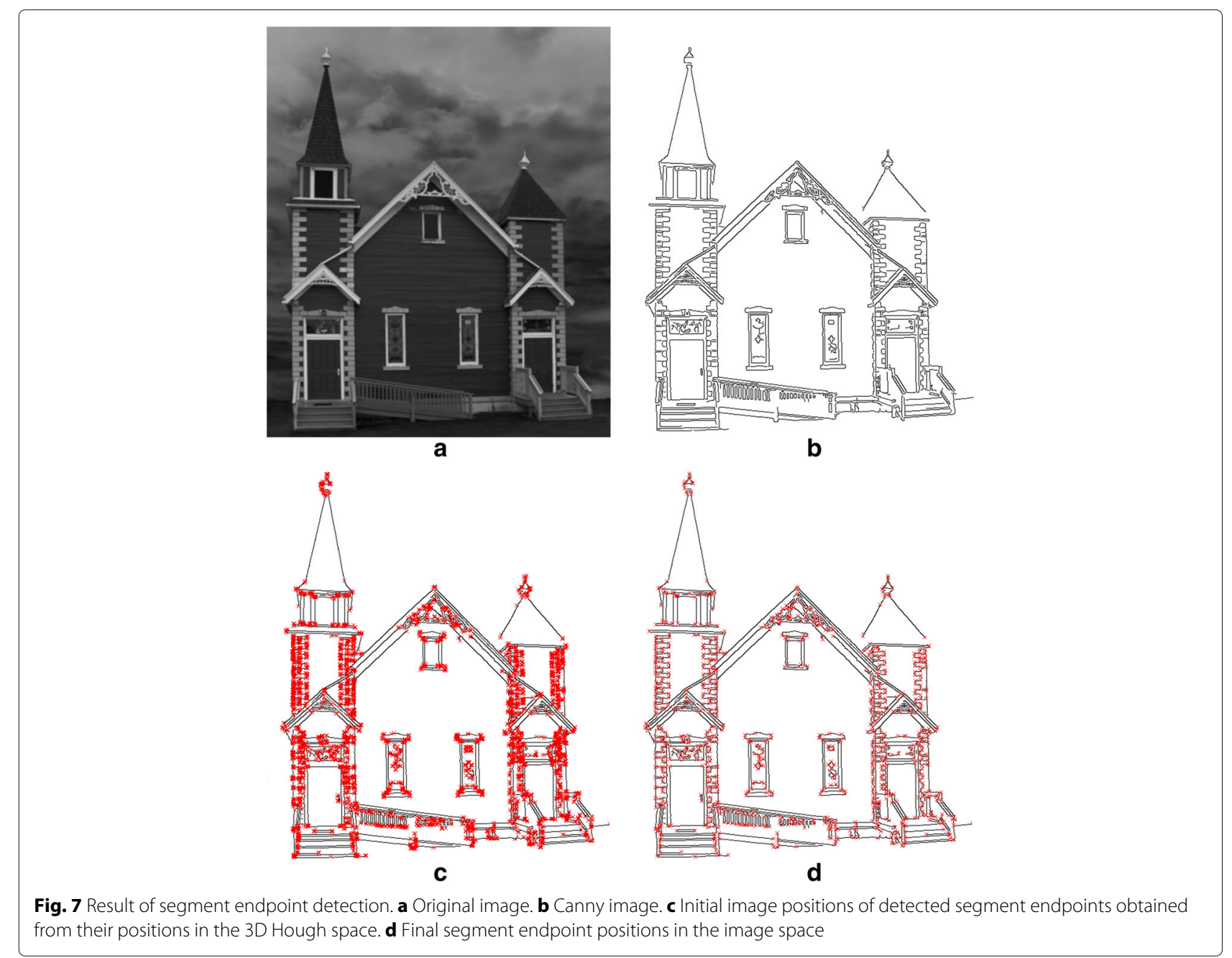



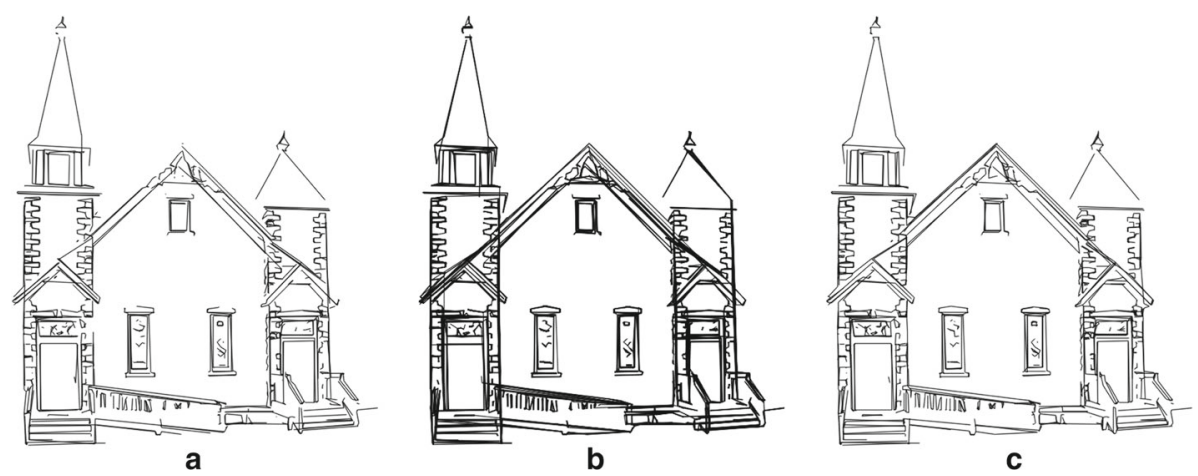

Fig. 8 Results of segment detection of the image in Fig. 7a. a Detected segments without considering segment endpoints of neighboring lines. b Detected segments without non-maximum suppression. c Detected segments using endpoints of neighboring lines and non-maximum suppression

any two image points $\left(x_{1}, y_{1}\right)$ and $\left(x_{2}, y_{2}\right)$. Assuming that $d_{1}^{\prime}$ and $d_{2}^{\prime}$ are the positions in $d$ of these two points for an angle $\theta+\phi$, the differences in $d$ of $\left(x_{1}, y_{1}\right)$ and $\left(x_{2}, y_{2}\right)$ for the two angles are related as follows:

$$
\left(d_{1}^{\prime}-d_{2}^{\prime}\right)=\left(d_{1}-d_{2}\right) \cos (\phi)+\left(p_{1}-p_{2}\right) \sin (\phi)
$$

Given $d_{1}^{\prime}-d_{2}^{\prime} \geq 0$ and $0<\phi<\pi / 2$, Eq. 10 can be rewritten as:

$$
\left(d_{1}^{\prime}-d_{2}^{\prime}\right) \leq\left(d_{1}-d_{2}\right)+\left(p_{1}-p_{2}\right) \tan (\phi)
$$

Assuming $\left(d_{1}^{\prime}-d_{2}^{\prime}\right)-\left(d_{1}-d_{2}\right) \geq 0^{1}$, the last equation provides an expression of the maximum change of position in $d$ of the segment defined by $\left(x_{1}, y_{1}\right)$ and $\left(x_{2}, y_{2}\right)$ for an increase in angle $\phi$ (a similar expression can be obtained for changes in $p$ ):

$$
\left(d_{1}^{\prime}-d_{2}^{\prime}\right)-\left(d_{1}-d_{2}\right) \leq\left(p_{1}-p_{2}\right) \tan (\phi)
$$

In order to make this maximum change $(C d)$ only dependent on $\phi$, the maximum possible value of $\left(p_{1}-p_{2}\right)$ is considered. This value, for pieces of segment formed by $\eta$ cells in the Hough representation of corners and endpoints, can be set to $\eta \Delta p$. Thus, Eq. 12 can be rewritten as follows:

$$
C_{d}=(\eta \Delta p) \tan (\phi)
$$

Taking into account the rounding and aliasing effects of the coordinate transformation between image and parameter spaces, a constant value of 1 pixel for $C_{d}$ is considered, which leads to the following final expression for $\phi$ :

$$
\phi=\arctan \frac{1}{\eta \Delta p}
$$

This reduces the number of orientation planes that must be checked for corners and endpoints detection. In addition, since $\phi$ and $\Delta p$ are inversely related, the increase of computations in each orientation plane caused by a decrease of $\Delta p$ is compensated by a reduction of the num-
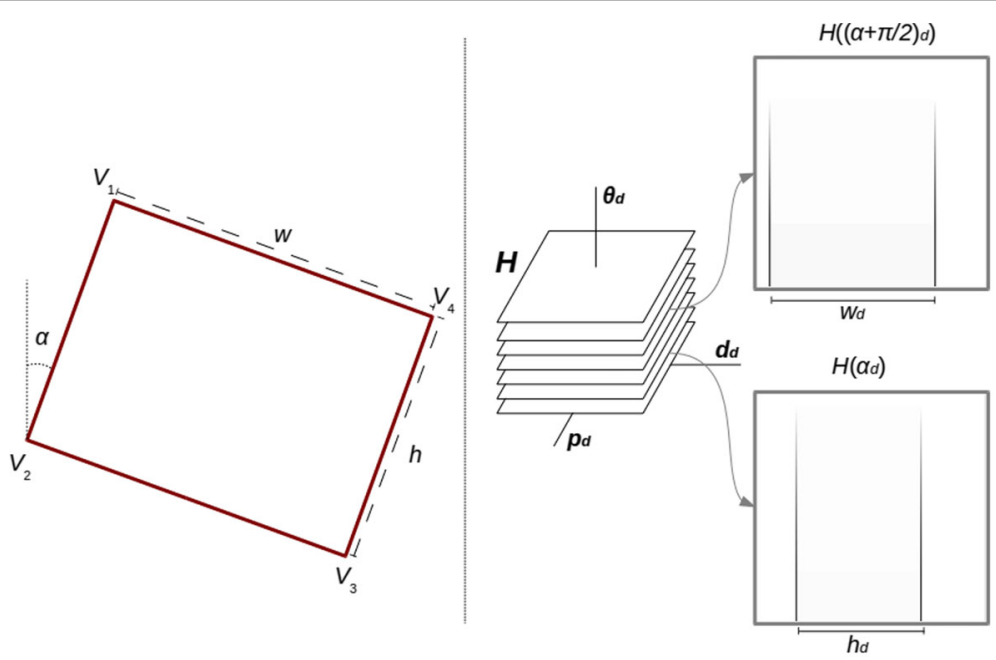

Fig. 9 Rectangle representation in the 3D Hough space 
ber of planes where the search must be done. Another important result of this definition for $\phi$ is that its value does not change with $\Delta \theta$. Thus, reducing the angular resolution does not increase the computational time of the detection process.

\subsubsection{Locating corners and endpoints in the image}

Once Hough cells containing corners and endpoints are detected, the next step is to compute their positions in the image. The set of image points belonging to a Hough cell $\left(H\left(\theta_{d}, d_{d}, p_{d}\right)\right)$ can be approximated using an image window of size $w \times w$, with $w$ the maximum between $\Delta d$ and $\Delta p$, centered on $\left(x_{c}, y_{c}\right)$ :

$$
\begin{aligned}
& x_{c}=d \cos (\theta)-p \sin (\theta) \\
& y_{c}=d \sin (\theta)+p \cos (\theta)
\end{aligned}
$$

with $\theta, d$, and $p$ being the real values associated with $\theta_{d}, d_{d}$, and $p_{d}$. Thus, given a Hough cell $\left(H\left(\theta_{d}, d_{d}, p_{d}\right)\right)$ containing a corner or endpoint, locating its image position can be solved by searching for the pixel within the corresponding image window that maximizes some corner/endpoint criterion. For this purpose, the minimum eigenvalue of the covariance matrix of gradients over the pixel neighborhood is used [29] ${ }^{2}$. Such a function provides maximum values at corners and endpoints in the point neighborhood.

Figure 7 shows the result of applying this process to a real image. Firstly, using the edge image of Fig. $7 \mathrm{~b}$,

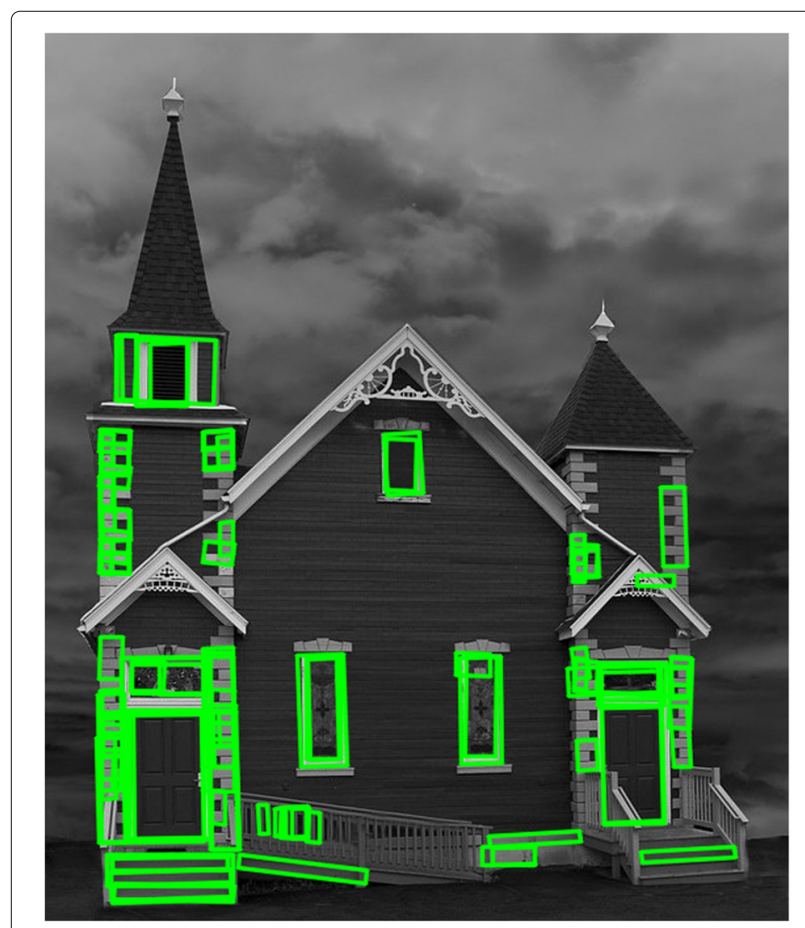

Fig. 10 Results of rectangle detection of the image in Fig. 7a the initial positions of corners/endpoints are obtained by detecting corner/endpoint patterns in the Hough space (Fig. 7c). Then, these positions are adjusted looking for the maximum of the minimum eigenvalue function in the pixel neighborhood (Fig. 7d).

\subsection{Detection of segments and polylines}

Once corners and endpoints have been extracted, image segments can be detected by checking the strength of any segment formed by a pair of points (Eq. 6). In order to avoid testing segments for every pair of points, detected segment endpoints are placed on the Hough space applying the corresponding transformation for every orientation value. This provides, for each line representation, a list of points with which segment validation should be done. Assuming that points on that list are ordered by the value of the parameter $p$, segment detection can be easily solved using Algorithm 3.

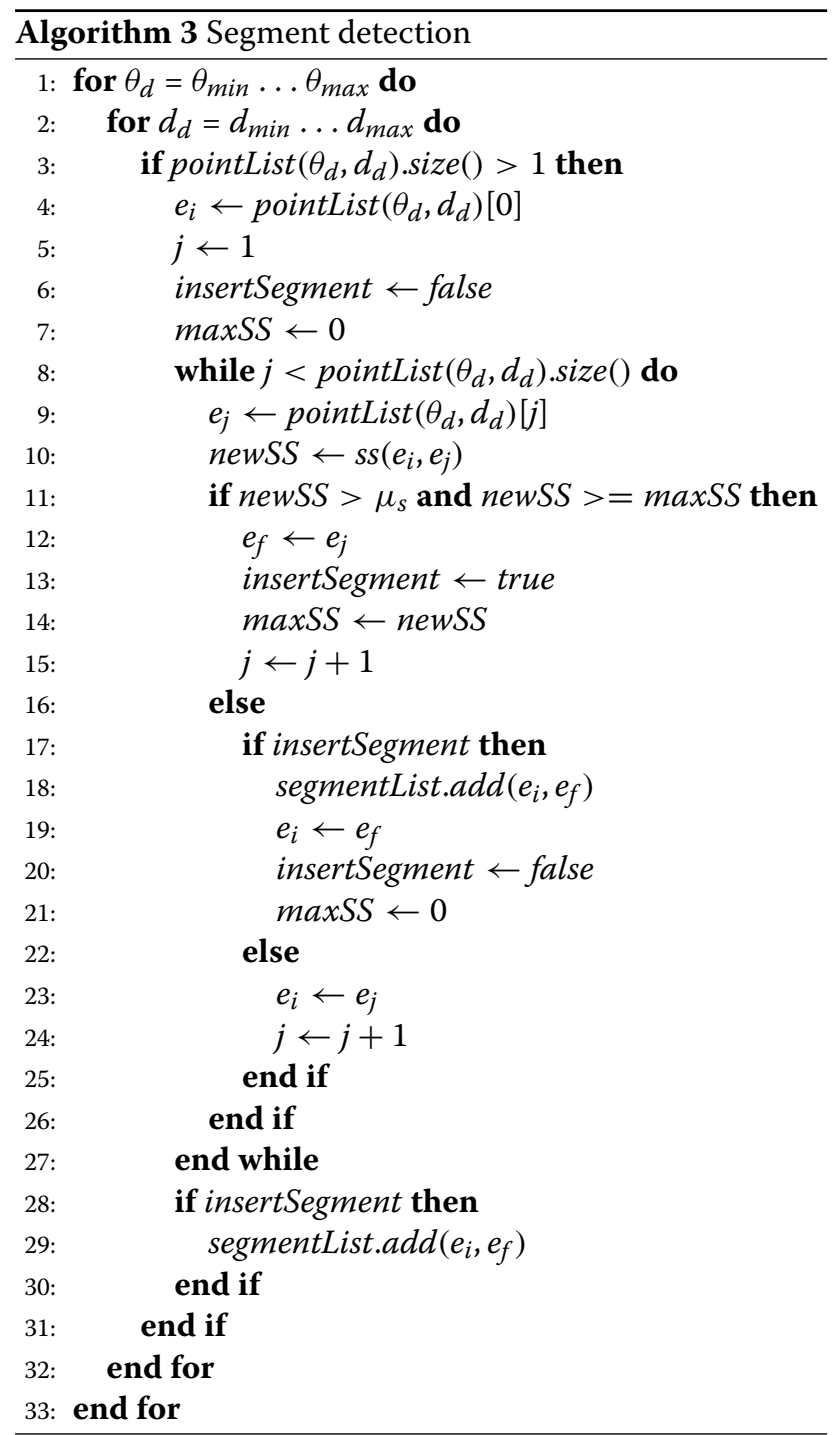


In this algorithm, pointList $\left(\theta_{d}, d_{d}\right)$ represents the aforementioned list of detected segment endpoints of the line defined by $\theta_{d}$ and $d_{d}$. If this list contains at least two points, a segment validation process begins. Given an initial segment endpoint (starting from the first element of the list), this process involves searching for the final endpoint of a common line segment (among the subsequent elements of the list) that produces the highest segment strength above a threshold $\mu_{s}$. This search stops if the current final endpoint provides a lower strength than the previous one. In such a case, the previous validated segment is stored and the process is restarted taking the last valid final endpoint as the initial endpoint of a new segment.

The rounding step of the coordinate transformation between image and Hough spaces could make an almost vertical segment divide into two pieces. To deal with this problem, if an image corner or endpoint falls near a cell of the neighboring line, it is included in the list of points of that line. This mostly solves the segment breakdown problem, although it may increase the number of false positives. Nevertheless, they can be discarded applying a subsequent non-maximum suppression step to the segments with a common endpoint. Thus, taking the angle defined by two segments with a common endpoint as a proximity measure, if two segments are considered near enough, the one with the smallest strength is removed from the set of detected segments.

Figure 8 shows the result of detecting the segments of image in Fig. 7a using the corners and endpoints of Fig. 7d. Figure 8a shows the result of segment detection without considering segment endpoints of neighboring lines. Figure $8 \mathrm{~b}$ shows the result of using endpoints close to each line without applying the non-maximum suppression process. Finally, Fig. 8c depicts detected segments using extra endpoints with non-maximum suppression.

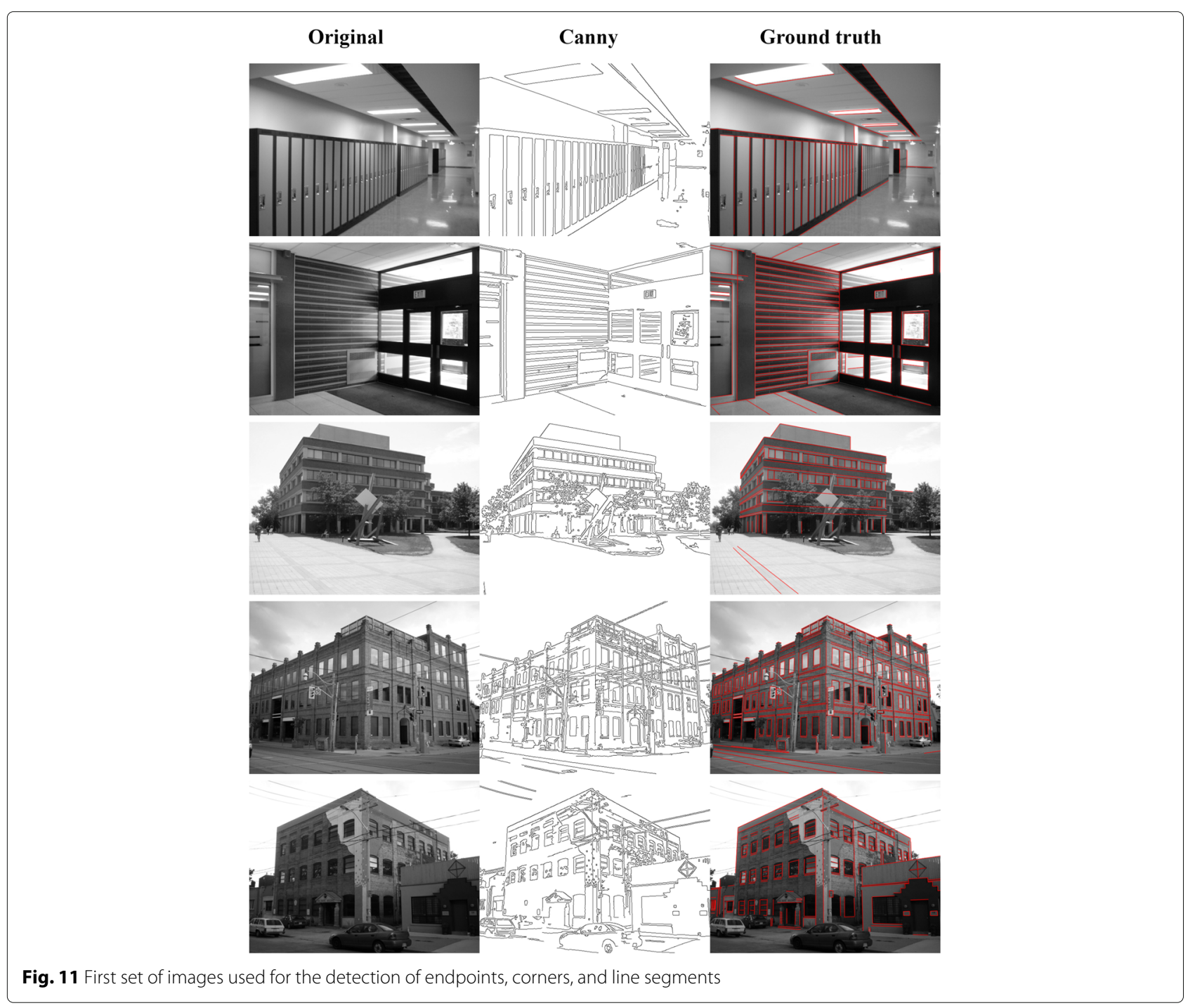


Since segments are confirmed from their endpoints, they are implicitly interconnected forming polylines. These polylines can be extracted from the graph representation that is obtained taking endpoints as vertices and segments as edges connecting vertices. Thus, each disconnected subgraph resulting from this representation corresponds to a polyline in the image. In the ideal case, when each node has one or two edges, this is the only valid interpretation of the graphs. However, if there exist vertices with more than two connections, each polyline can be decomposed into simple pieces. In such cases, a graph partitioning technique [30] can be applied in order to obtain a more realistic polyline representation.

\subsection{Computational complexity of the corner and segment detection method}

The analysis of the computational complexity of the proposed corner and segment detection method can be studied by considering its five different phases: (a) first stage of the voting process (line voting); (b) second stage of the voting process (accumulation); (c) corner/endpoint detection; (d) corner/endpoint mapping between image and Hough spaces; and (e) segment detection. Assuming the Hough space is formed by $l$ orientation planes of size $m \times m$ and taking $n$ as the number of edge points, the two stages of the voting process can be performed with computational costs $O(m n)$ and $O\left(l m^{2}\right)$, respectively. Using a single voting process would entail a computational cost of $O(l m n)$ instead. It must be noted that generally $n$ is much bigger than $m$, so in practice, the two-stage voting scheme is much less time-consuming. Corner detection is solved with a computational cost of $O\left(l_{r} m^{2}\right)$, with $l_{r}$ being the number of orientation planes using $\phi$ (Eq. 14) as angle resolution. $l_{r}$ is significantly smaller than any of the dimensions of the Hough space, so this computational cost can be considered sub-cubic. Taking $p$ as the

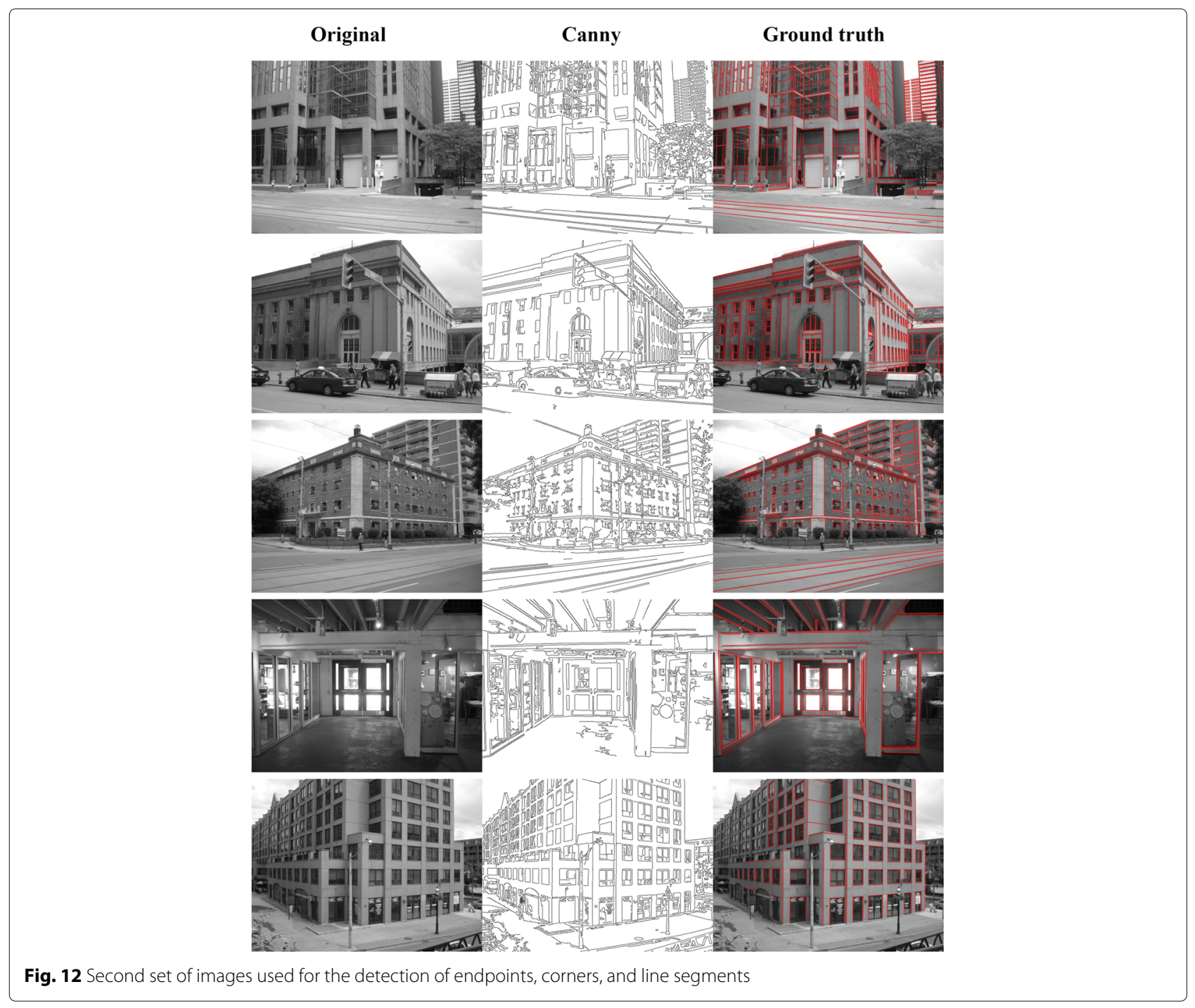


number of detected corners/endpoints, the mapping of these points from the image space to the Hough space, as a previous step to segment detection, is achieved with cost $O(l p)$. This is also the computational cost of segment detection, since each plane will contain $p$ points and each point can at most confirm two segments in the same orientation plane.

Despite all the included optimizations, the proposed method presents a clearly higher computational cost than many existing approaches for independent detection of corners and line segments. Nevertheless, all its phases iteratively carry out the same processing over independent elements of both image and Hough spaces, which makes them inherently parallelizable. Taking advantage of this, we have developed a parallel implementation of the proposed corner and segment detector on a GPU. This implementation attempts to approximate the computational cost of each phase to linear time in relation to the image size. Regarding the sequential version, speedups above $400 \times$ in some of the phases and about $35 \times$ in the whole method, including data exchanges between CPU and GPU, have been obtained ${ }^{3}$. Although this implementation significantly outperforms its sequential counterpart, we are working on new optimizations to improve the speedup dealing with GPU programming issues such as coalesce memory accesses and optimal occupancy.

\subsection{Detection of other image features}

As explained above, the segment detection process provides not only a set of segments but also a set of polygonal chains. These polygonal chains have arbitrary shapes since they are the result of the implicit connection of the detected segments. Nevertheless, the proposed Hough space can also be used for the detection of predefined polygonal shapes. One of the simplest cases is the detection of rectangles $[31,32]$.

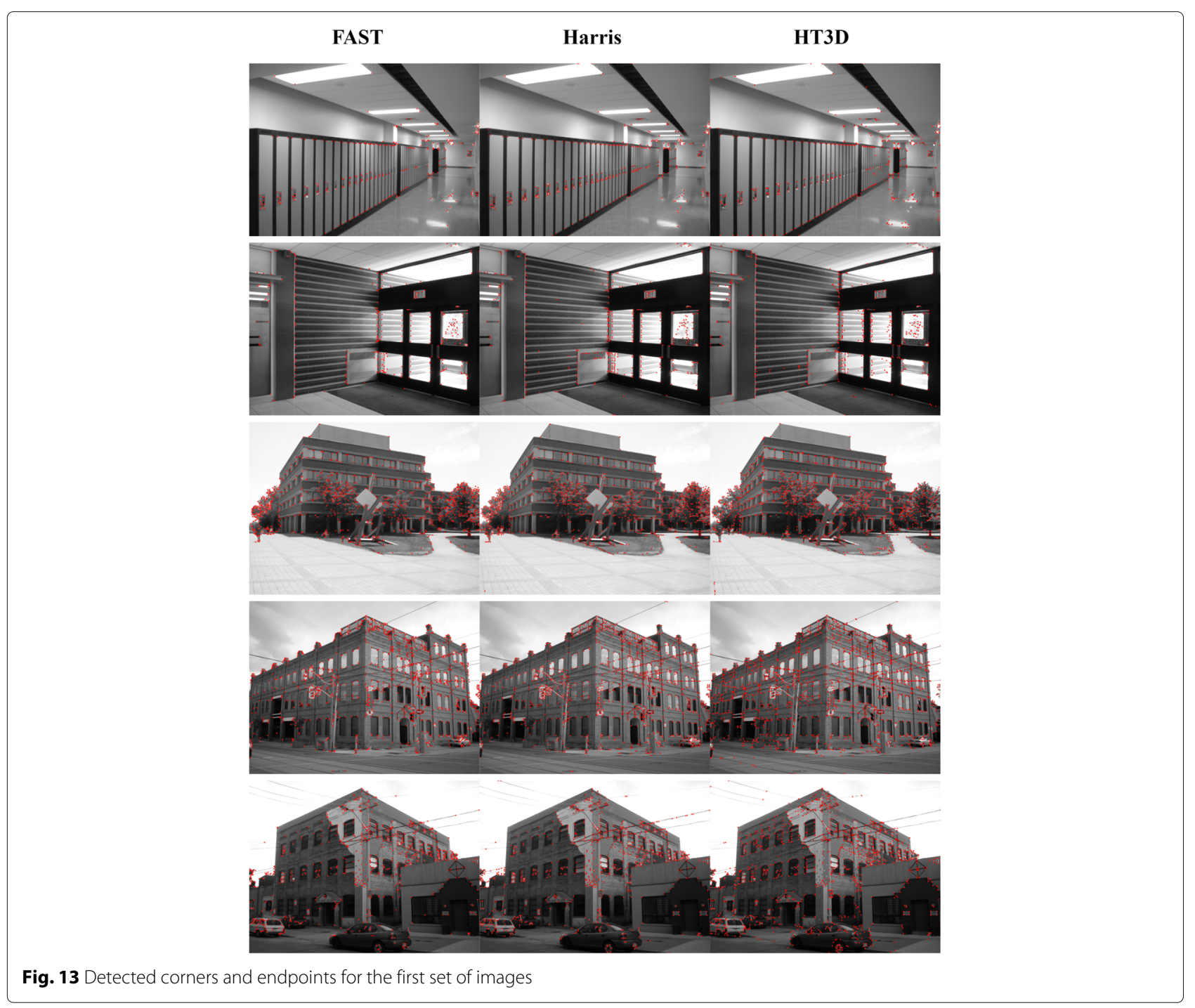


Since a rectangle is composed of four segments, the 3D Hough space makes it possible to compute the total number of points included in the contour of the rectangle. Thus, considering a rectangle expressed by its four vertices $V_{1}=\left(x_{1}, y_{1}\right), V_{2}=\left(x_{2}, y_{2}\right), V_{3}=\left(x_{3}, y_{3}\right)$, and $V_{4}=\left(x_{4}, y_{4}\right)$ (see Fig. 9), the number of points of its contour, denoted as $H_{r}$, can be computed as:

$$
H_{r}=H_{1 \leftrightarrow 2}+H_{2 \leftrightarrow 3}+H_{3 \leftrightarrow 4}+H_{4 \leftrightarrow 1}
$$

where each $H_{i \leftrightarrow j}$ denotes the number of points of the segment defined by $V_{i}$ and $V_{j}$ (Eq. 5).

Figure 9 shows an example of rectangle representation in the discrete parameter space. Each pair of parallel segments of the rectangle is represented in the corresponding orientation plane of the Hough space: $H\left(\alpha_{d}\right)$ for one pair of segments and $H\left((\alpha+\pi / 2)_{d}\right)$ for the other one, with $\alpha_{d}$ and $(\alpha+\pi / 2)_{d}$ being the discrete values associated to $\alpha$ (rectangle orientation) and $(\alpha+\pi / 2)$, respectively.
For each orientation plane, there is a representation of how many points contribute to each cell $\left(d_{d}, p_{d}\right)$, i.e., how many points belong to every segment of the corresponding orientation. A high histogram contribution is represented in the figure with a dark gray level, while a low contribution is depicted with an almost white color. As it can be observed, the highest contributions are found in parallel segments with displacements of $w_{d}$ and $h_{d}$, which are the discrete values associated to the rectangle dimensions.

Taking this rectangle representation into account and considering continuous values of each parameter for simplification, each $H_{i \leftrightarrow j}$ of expression 17 can be rewritten as follows:

$$
\begin{aligned}
H_{1 \leftrightarrow 2}= & \left|H\left(\alpha, d_{1 \leftrightarrow 2}, d_{4 \leftrightarrow 1}\right)-H\left(\alpha, d_{1 \leftrightarrow 2}, d_{2 \leftrightarrow 3}\right)\right| \\
H_{2 \leftrightarrow 3}= & \mid H\left(\alpha+\pi / 2, d_{2 \leftrightarrow 3},-d_{1 \leftrightarrow 2}\right) \\
& -H\left(\alpha+\pi / 2, d_{2 \leftrightarrow 3},-d_{3 \leftrightarrow 4}\right) \mid
\end{aligned}
$$

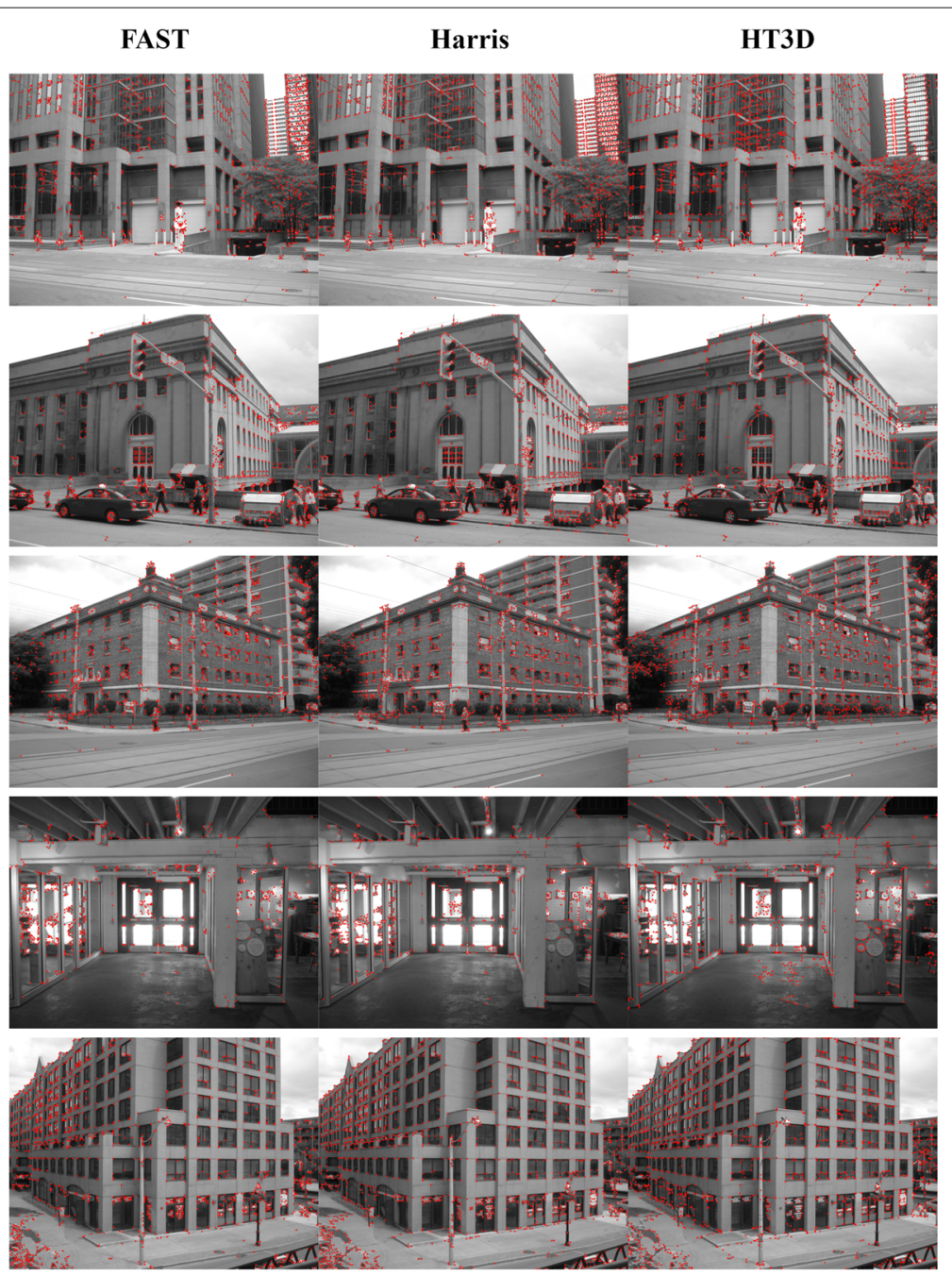

Fig. 14 Detected corners and endpoints for the second set of images 


$$
\begin{aligned}
H_{3 \leftrightarrow 4}= & \left|H\left(\alpha, d_{3 \leftrightarrow 4}, d_{2 \leftrightarrow 3}\right)-H\left(\alpha, d_{3 \leftrightarrow 4}, d_{4 \leftrightarrow 1}\right)\right| \\
H_{4 \leftrightarrow 1}= & \mid H\left(\alpha+\pi / 2, d_{4 \leftrightarrow 1},-d_{3 \leftrightarrow 4}\right) \\
& -H\left(\alpha+\pi / 2, d_{4 \leftrightarrow 1},-d_{1 \leftrightarrow 2}\right) \mid
\end{aligned}
$$

with $d_{i \leftrightarrow j}$ being the parameter $d$ of the straight line defined by the points $V_{i}$ and $V_{j}$, which are related with the rectangle dimensions as follows

$$
w=d_{3 \leftrightarrow 4}-d_{1 \leftrightarrow 2}
$$

$$
h=d_{2 \leftrightarrow 3}-d_{4 \leftrightarrow 1}
$$

According to these expressions, rectangle detection can be solved by searching for those combinations of $\left(\alpha, d_{1 \leftrightarrow 2}, d_{2 \leftrightarrow 3}, d_{3 \leftrightarrow 4}, d_{4 \leftrightarrow 1}\right)$ for which $H_{r} /(2 * w+2 * h)>$ $\tau_{r}$, given a certain value of $\tau_{r}$ near to 1 . To improve the efficiency of this process, instead of checking each combination of these parameters, previously detected segment endpoints can be used to preselect an initial subset of

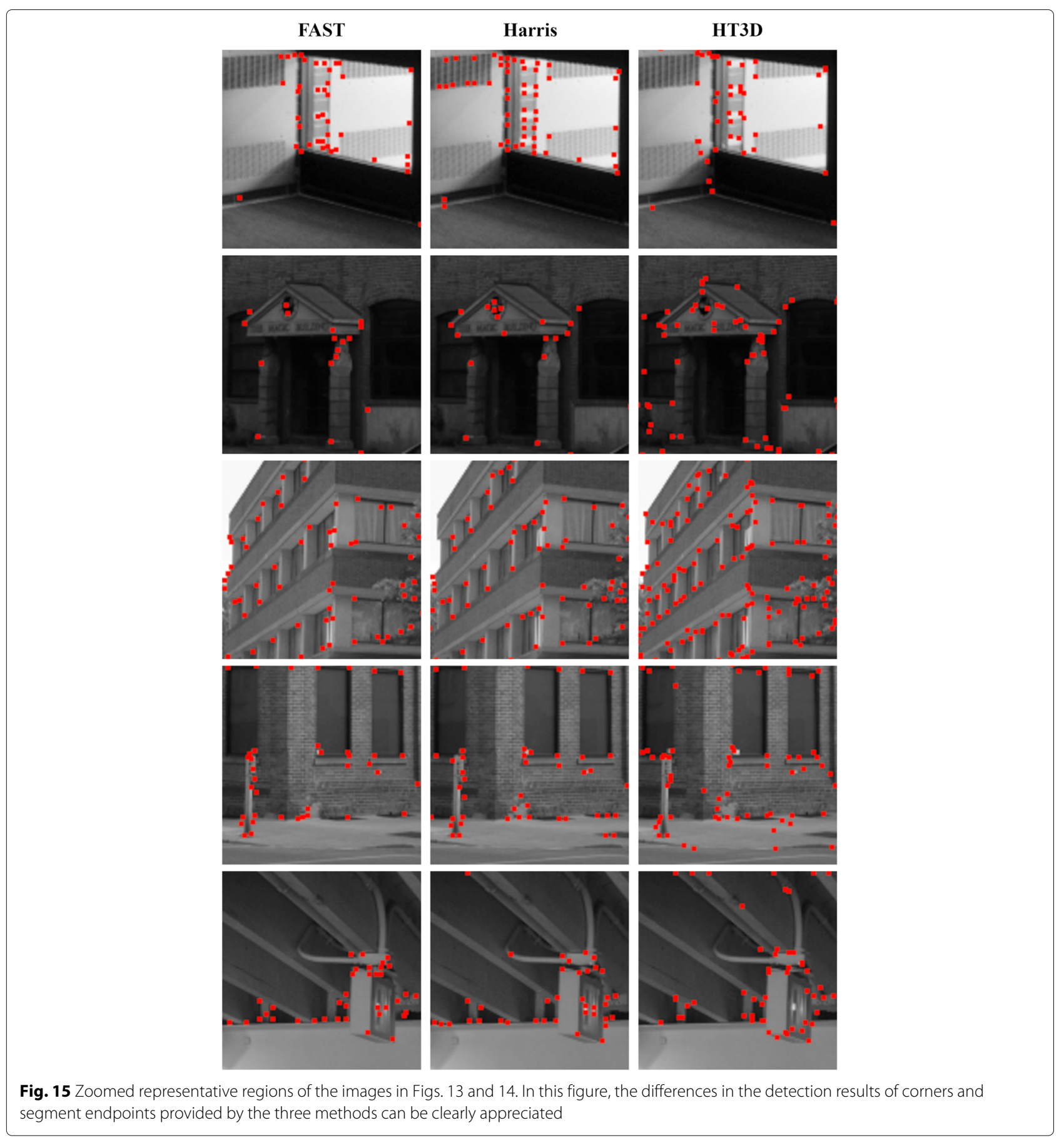


Table 1 Number of detected corners/endpoints $\left(N E p_{d}\right)$ for the ten test images

\begin{tabular}{llll}
\hline & \multicolumn{3}{c}{$N E p_{d}$} \\
\cline { 2 - 4 } & FAST & Harris & HT3D \\
\hline 1 & 273 & 330 & 375 \\
3 & 318 & 401 & 477 \\
4 & 1084 & 1165 & 1369 \\
5 & 1306 & 1315 & 1753 \\
6 & 833 & 910 & 1336 \\
7 & 1400 & 1662 & 1984 \\
8 & 1139 & 1264 & 1341 \\
9 & 1395 & 1561 & 1866 \\
10 & 799 & 846 & 1108 \\
\hline
\end{tabular}

combinations. Thus, a similar procedure to the one shown in Algorithm 3 can be applied for $\theta<\pi / 2$ to find the first segment of a potential rectangle, which provides values for $\alpha, d_{1 \leftrightarrow 2}, d_{2 \leftrightarrow 3}$ and $d_{4 \leftrightarrow 1}$. Then, using the list of points of the Hough line corresponding to $l\left(d_{2 \leftrightarrow 3}, \alpha+\pi / 2\right)$, possible values for $d_{3 \leftrightarrow 4}$ are obtained, completing the quintuple defining a rectangle.

Figure 10 shows the result of applying the above process to the image in Fig. 7a. During the detection process, the segment endpoints of Fig. $7 \mathrm{~d}$ are used for restricting the parameters of potential rectangles as previously described.

\section{Results and discussion}

To evaluate the performance of the proposed detection methods, they have been applied to a set of real images with ground truth. This section shows the results, comparing them with the ones obtained using other approaches.

A critical point when using an HT-based approach is to decide the resolutions of the parameter space. Finer quantizations do not always provide better results. Indeed, noise sensitivity grows for higher precision with regard to $d$ [33]. Zhang [34] proposes determining $\Delta d$ by considering the digitization of the spatial domain and then computes $\Delta \theta$ using the following relation:

$$
n_{d}=\frac{l \Delta \theta}{2 \Delta d}
$$

with $n_{d}$ within the interval $[0.5,2]$ and $l$ the length of the longest line segment.

Fixing a value for $\Delta d$ (and also for $\Delta p$ ) depends not only on the need for a detailed result but mainly on the nature of the image. Thus, for a noisy image, the detection method will perform better using a value of $\Delta d$ that is not too small. In addition, if the image complexity is not too high, understanding complexity in terms of the number and distribution of edge point chains, a low value of $\Delta d$ will provide a similar result to a higher one. For the test images used to evaluate our proposal, a value of 2 for $\Delta d$ and $\Delta p$ has been chosen. However, it must be pointed out that, for some of them, higher resolutions produce a comparable detection rate. $\Delta \theta$ has been fixed using expression 24 with $n_{d}=1$ and $l=400$.

Results for corner and segment detection have been obtained using the YorkUrbanDB dataset [35]. This dataset contains 102 marked images of urban environments. Each image is labeled to identify the subset of line segments that satisfy the Manhattan assumption [36]. Both quantitative and qualitative evaluations have been carried out for the whole set of images, comparing our corner and segment detector (HT3D) with two corner

Table 2 Comparison of detected corners/endpoints with the ground truth for the ten test images

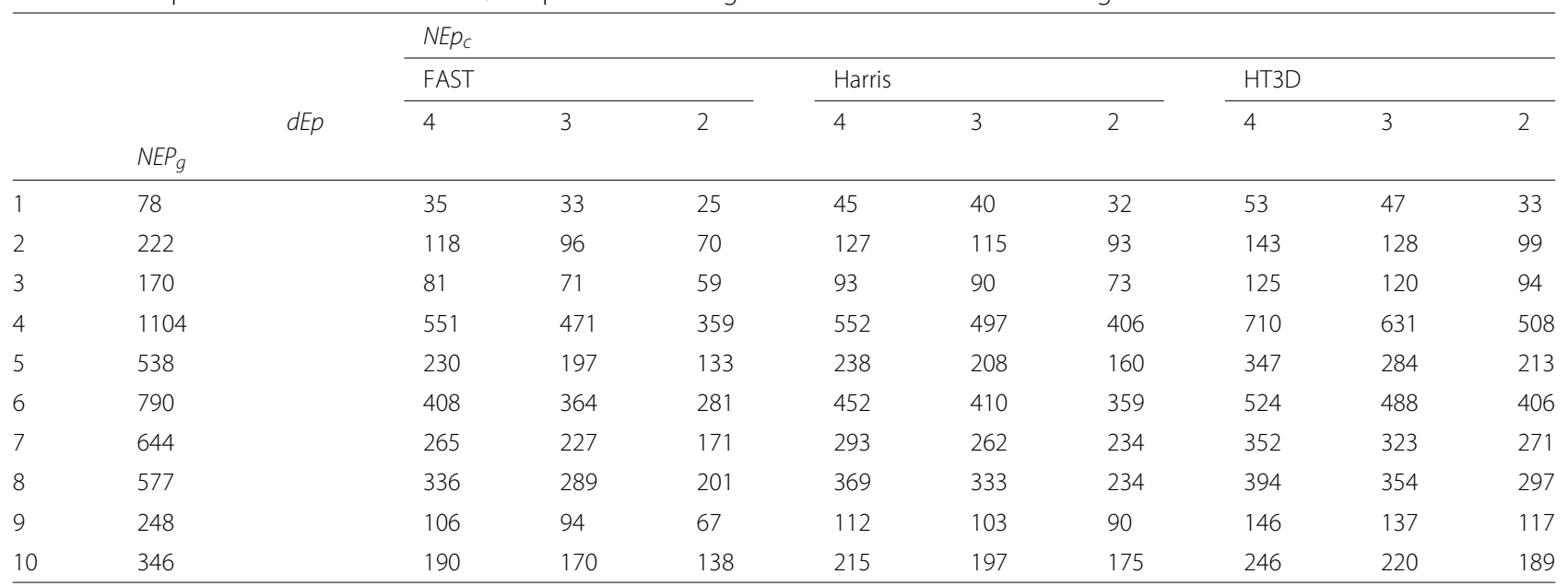

The number of correct matchings of each method $\left(N E p_{c}\right)$ is shown considering different values of the distance between ground truth and detected points $(d E p)$. The total number of ground truth points $\left(N E P_{g}\right)$ is shown in the first column 


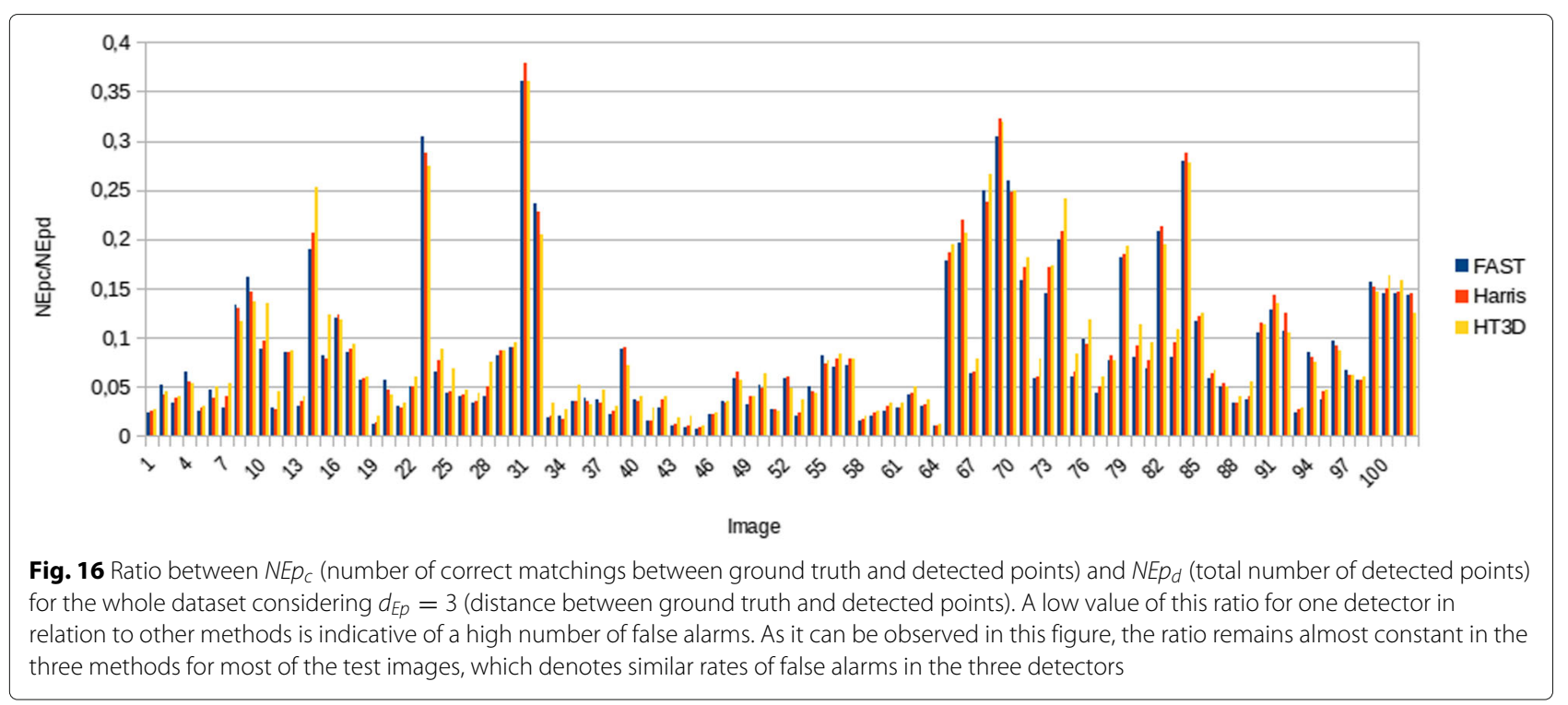

detectors and two segment detectors. A quantitative comparison of the different approaches using the 102 images composing the dataset is shown here. Complete results for a subset of ten images are also included. This subset corresponds to the ten scenes of Figs. 11 and 12. For each scene, these figures show the original image, the Canny image, used as input in our approach, and the ground truth. Every image has been smoothed using a $5 \times 5$ Gaussian filter with a standard deviation of 1 before being applied to each method, except for one of the segment detectors (LSD), since it includes its own smoothing step.

For corner and endpoint detection, our method has been compared with Harris [37] and FAST [38].
Parameters of these methods have been experimentally chosen trying to favor the detection results as much as possible according to the relation between true and false corners. Specifically, for the Harris method, a neighborhood size of 5 pixels and a sensitivity factor of 0.04 have been used. Likewise, the intensity comparison threshold of FAST has been set to 15 . In the proposed method, a length of 4 cells has been taken for the pieces of segments defining corners and endpoints in the Hough space (parameter $\eta$ ). Also, the angle range to confirm corners has been set to $\left[75^{\circ}, 105^{\circ}\right]$.

Figures 13 and 14 show the corner/endpoint detection results for the two sets of images in Figs. 11 and 12

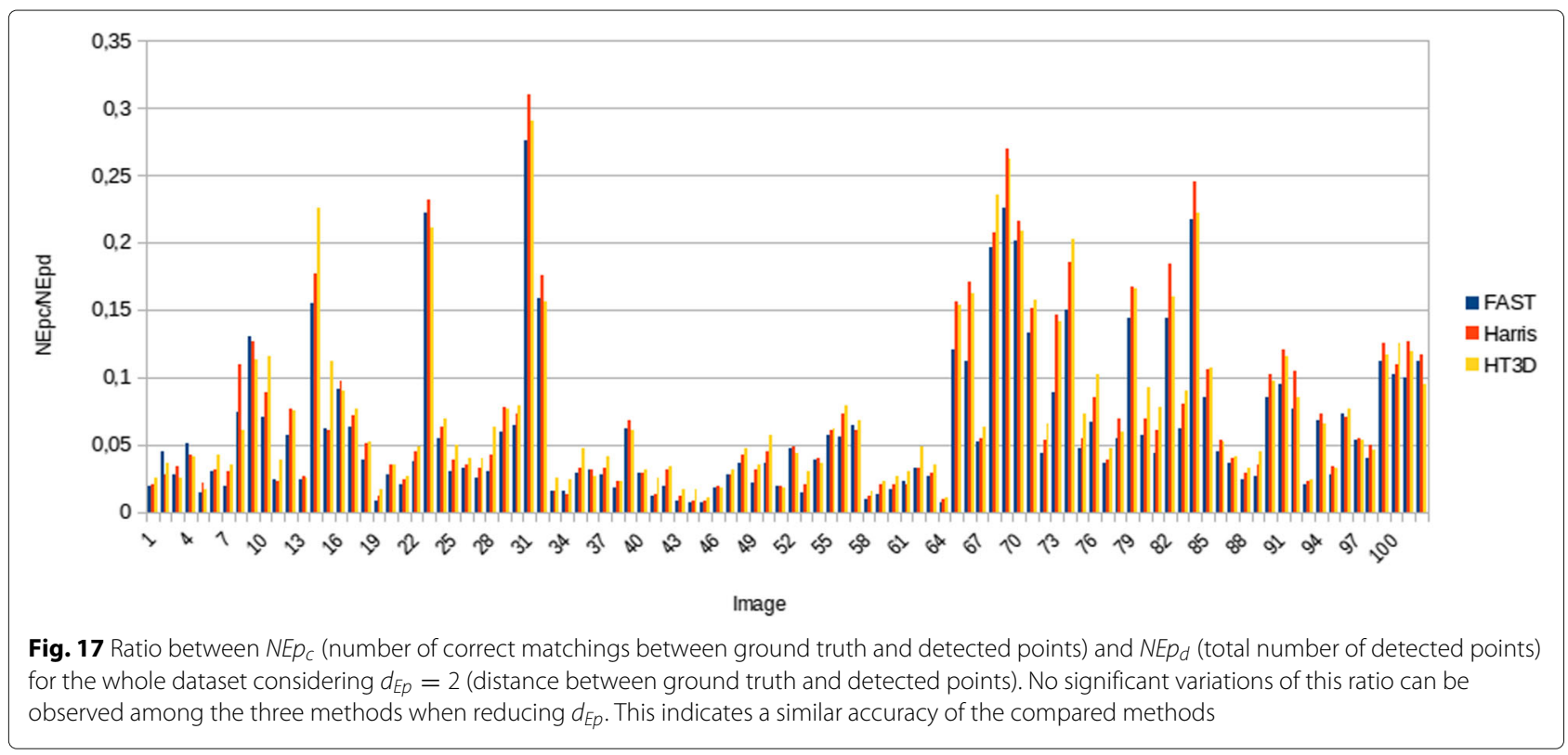




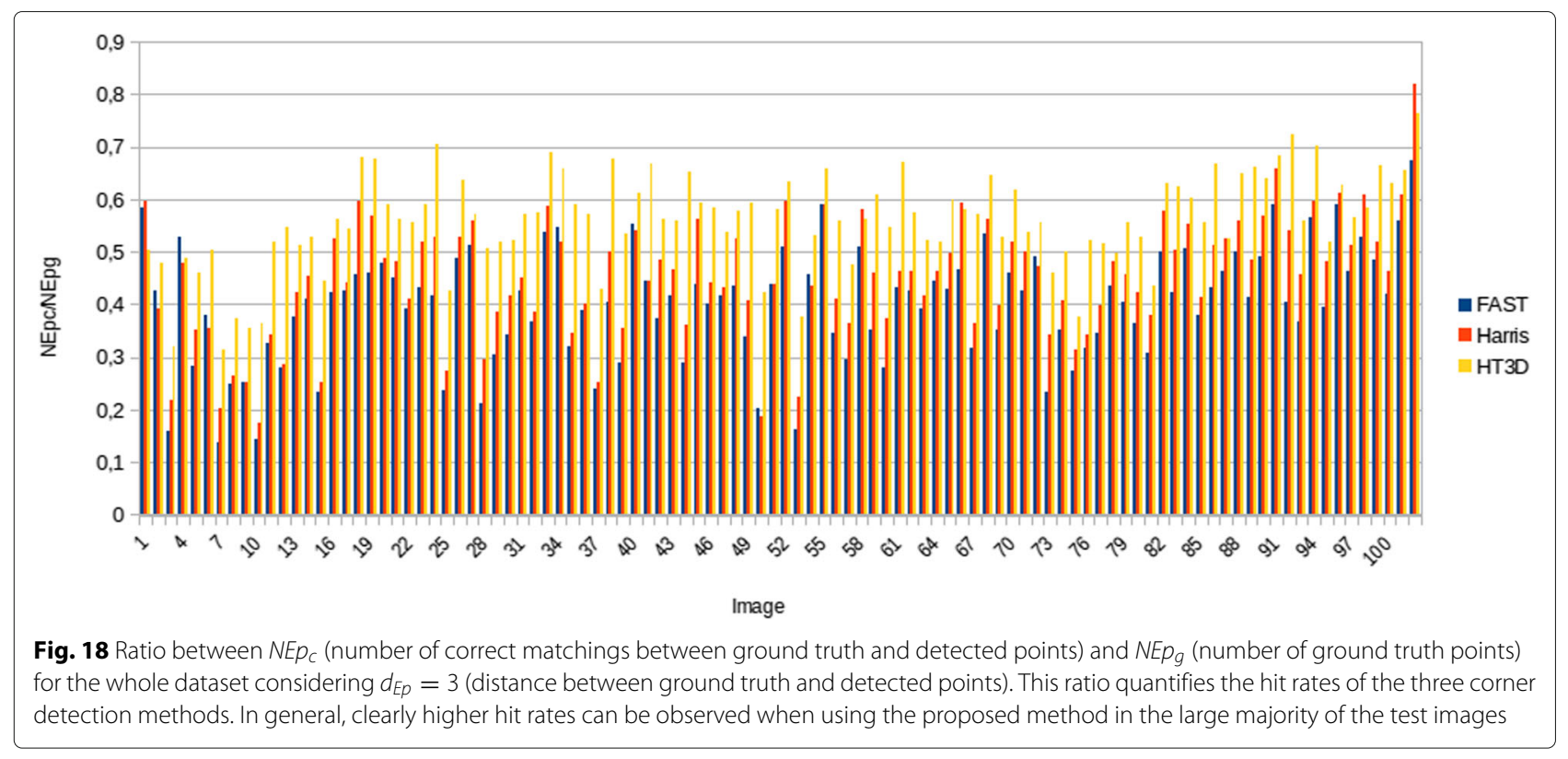

using FAST, Harris, and the proposed method. Figure 15 shows some representative regions of these images where the differences among the detection results of the compared approaches can be clearly appreciated. As it can be observed, the three methods perform well in the detection of corners resulting from the intersection of straight perpendicular edges. However, in most cases, the proposed method provides additional corner points. Some of them correspond to corners with non-right angles, mainly obtuse-angle corners, and to corners with low intensity in one of its edges (see the first three rows of images in Fig. 15). In addition, as it was expected, only our method detects non-intersection segment endpoints (see the last two rows of Fig. 15). They can be mostly found in the intersections between image lines and image limits. All these additional distinctive points are essential for line segment detection.

In order to quantitatively evaluate the three methods, each group of detected points has been compared with the segment endpoints of the ground truth data. Three

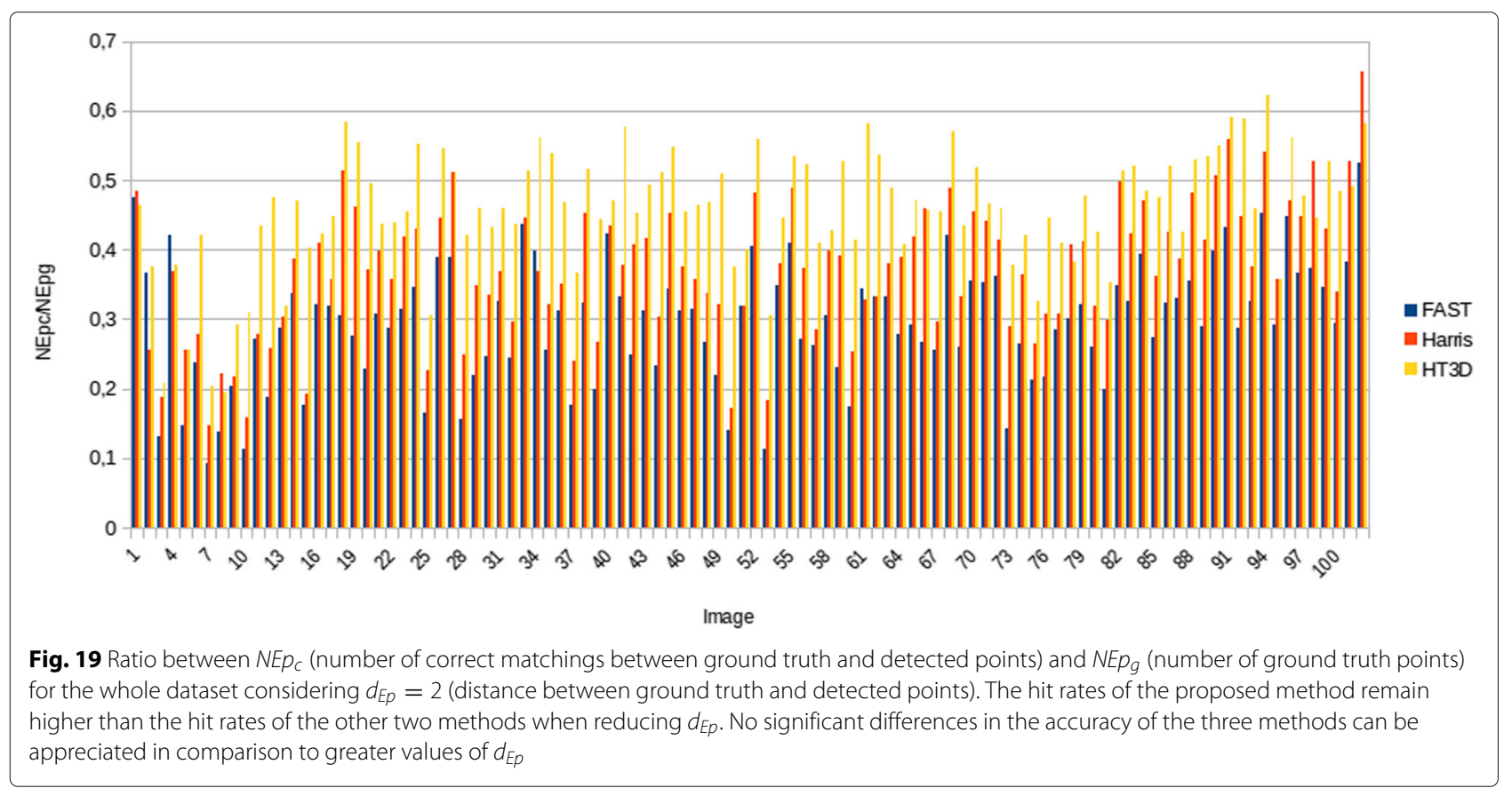


measures were considered in this comparison: number of detected corners/endpoints $\left(N E p_{d}\right)$, number of points of the ground truth data $\left(N E p_{g}\right)$, and number of correct matchings between ground truth and detected points $\left(N E p_{c}\right)$. To compute $N E p_{c}$, different values of the maximum euclidean distance $(d E p)$ between detected and ground truth points were considered. Tables 1 and 2 show the results obtained for the ten test images. Table 1 contains the total number of detected corners/endpoints using each method. Table 2 shows the number of ground truth points of each image and the number of correct matchings of the different detectors taking values of 4 , 3 , and 2 for $d E p$. Results show greater values of $N E p_{d}$ in HT3D than in FAST and Harris, although $N E p_{c}$ grows linearly. This behavior can also be observed in the entire dataset from the results depicted in Figs. 16 and 17. These figures show the ratio between $N E p_{c}$ and $N E p_{d}$ for each image of the dataset considering values for $d E p$ of 3 and
2 , respectively. Since the ground truth only includes a subset of potential line segments, false alarm rates cannot be directly measured. Nevertheless, the ratio between the number of correct matchings and the total number of detected features constitutes a false alarm indicator in the comparison among different methods. Thus, if a method presents a clearly low value of this ratio in relation to other approaches, it can be stated that the number of false detections of that method is significantly higher. For the present comparison among the corner/endpoint detectors, as it can be seen in Figs. 16 and 17, the ratio remains almost constant in the three methods for all the tests, which denotes similar rates of false detections in all of them. In relation to the hit rate of each approach, which has been measured as the ratio between the number of correct matchings and the number of ground truth points, Figs. 18 and 19 show higher values for HT3D than for the other two methods, regardless of the value of $d E p$. Thus, on the

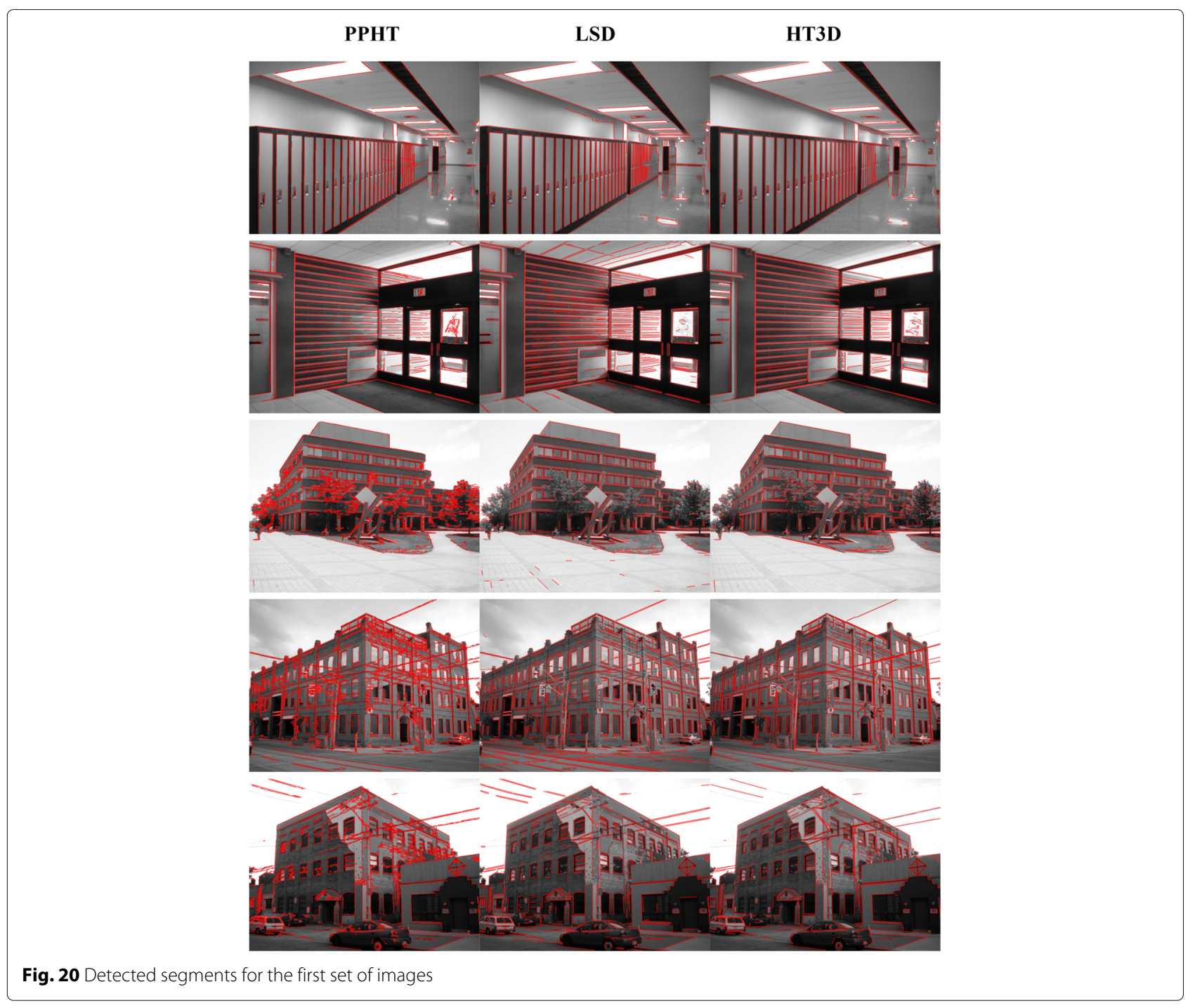


basis of this ratio, HT3D performs better than FAST in almost $100 \%$ of the images and better than Harris in about $95 \%$ of the tests. Since the difference among the hit rates of the three detectors does not significantly vary when reducing $d E p$, the accuracy provided by all the methods can be considered comparable.

Regarding segment detection, our method has been compared with two segment detectors: PPHT [19] (see Section 1 for a brief description) and LSD [39], a nonHough-based line segment detector, which works without parameter tuning. In PPHT, the parameter space has been discretized using values of 1 and $\pi / 180$ for distance and angle resolutions. Also, a minimum number of line votes of 20 and a value of 5 for the minimum segment length and the maximum gap of points of the same line have been used. In our method, segments have been detected considering a minimum segment strength of 0.8. Results of both Hough-based methods have been obtained from the same edge images.
The detected segments using the three methods for the ten test images are displayed in Figs. 20 and 21. Some zoomed representative regions of these images are shown in Fig. 22. The three segment detectors have also been quantitatively evaluated considering, firstly, the number of detected segments $\left(N S_{d}\right)$ and the percentage of connected segments $(C S)$. In images of real environments, segments do not usually appear as isolated entities, but as part of more complex features that allow object contours to be described. This is especially true in man-made environments, where, for instance, windows, doors, and walls are composed of sets of segments which are connected forming polylines. In this regard, we believe that the ability of a segment detector to obtain not individual segments but chains of segments makes the detection results more reliable. Table 3 shows a quantization of the segment-connectivity ability of the three methods through the measurement CS. As it can be observed, PPHT, with the chosen parametrization, does not provide

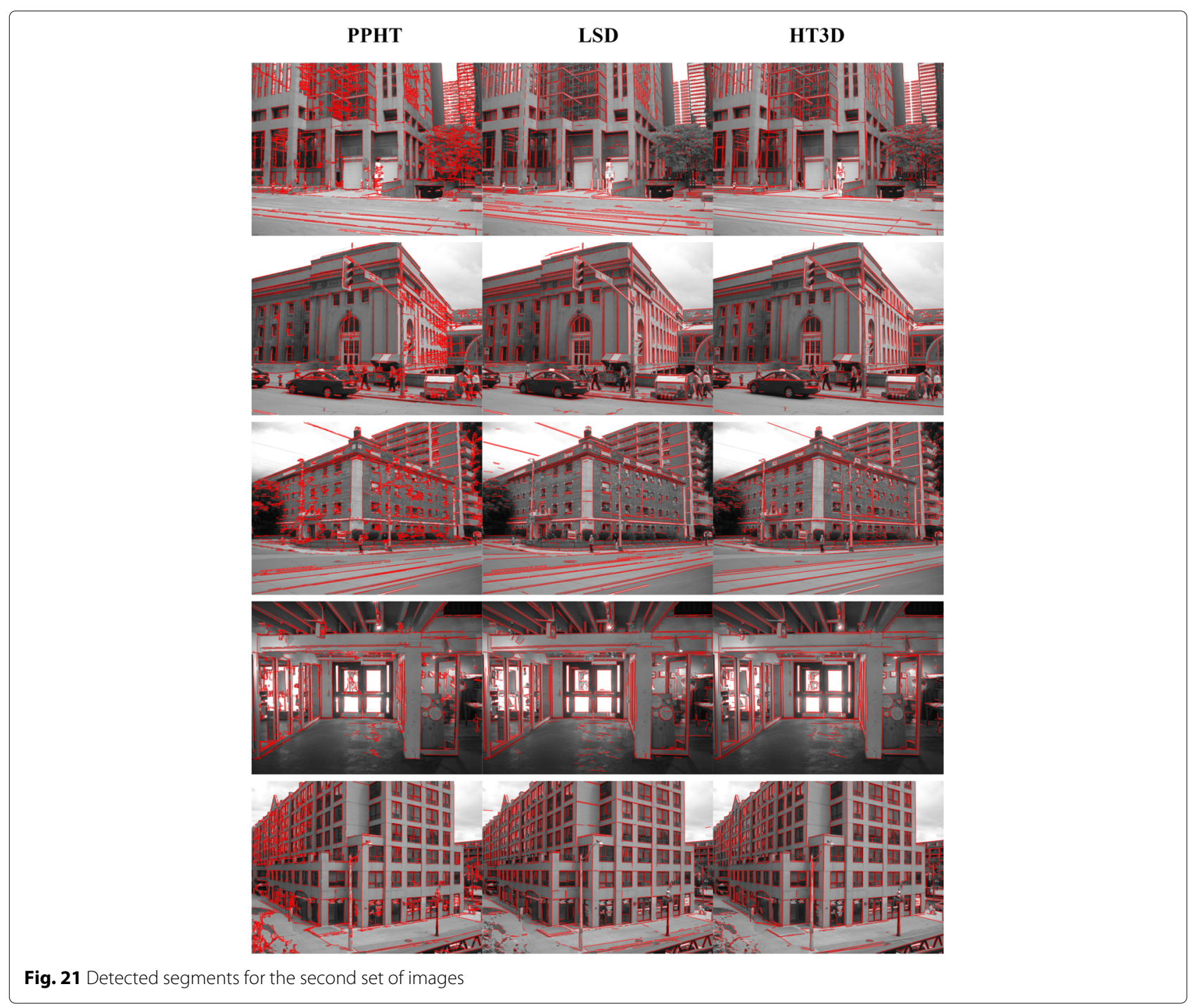




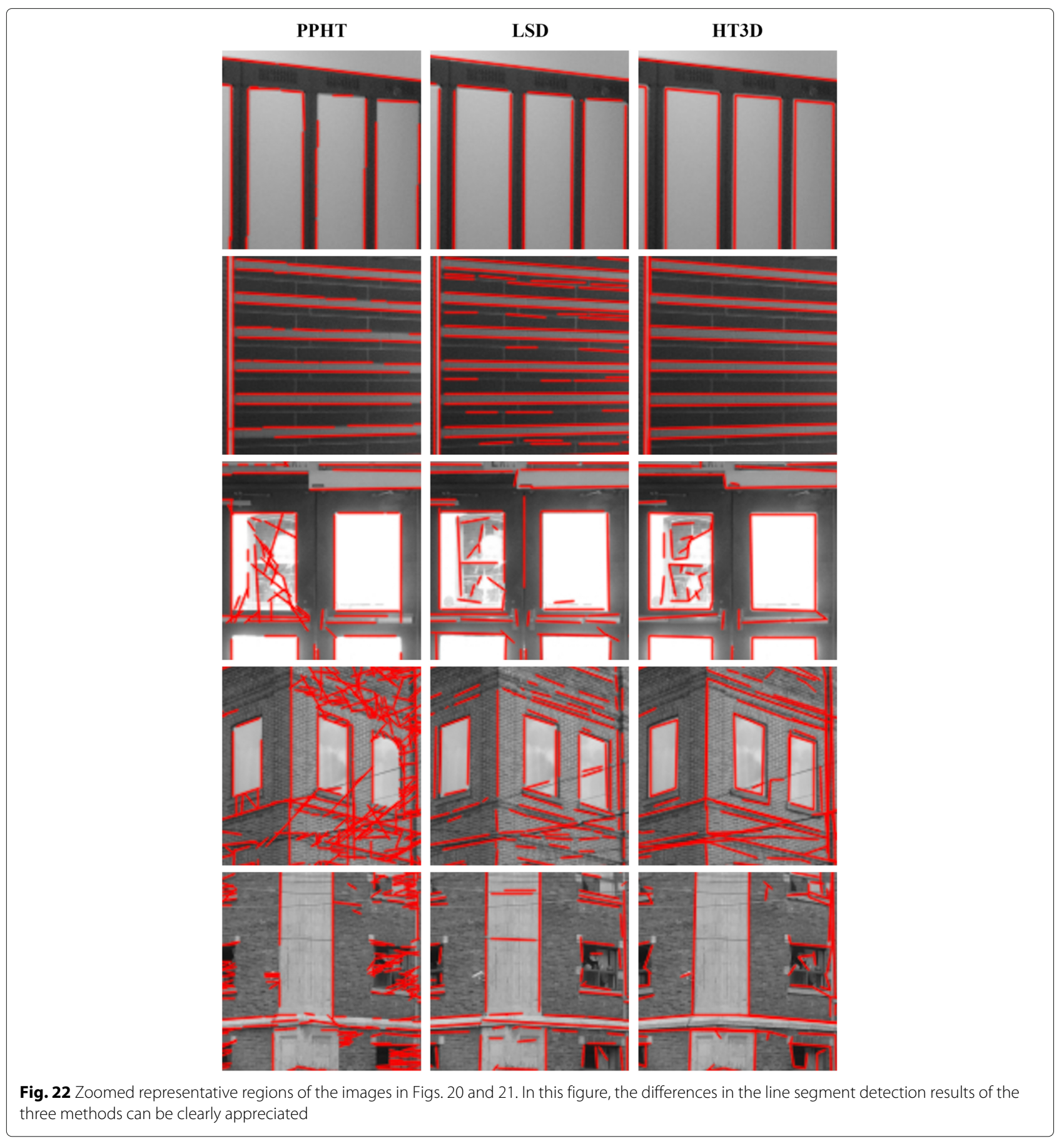

any connections among detected segments for any of the test images. Detected segments from LSD do present some degree of connectivity, but the connectivity rate is much lower than the one obtained by HT3D, which provides results in greater accordance with the kind of scenes in the image set.

Results of the three segment detector have also been compared with the ground truth data, using the Hausdorff distance [40] to determine the correct matchings. Given two segments $s_{i}=\left(e_{i 1}, e_{i 2}\right)$ and $s_{j}=\left(e_{j 1}, e_{j 2}\right)$, the Hausdorff distance between $s_{i}$ and $s_{j}$, denoted as $h d\left(s_{i}, s_{j}\right)$, is defined as follows:

$$
\begin{aligned}
h d\left(s_{i}, s_{j}\right)= & \min \left(\max \left(\left|e_{i 1}-e_{j 1}\right|,\left|e_{i 2}-e_{j 2}\right|\right),\right. \\
& \left.\max \left(\left|e_{i 1}-e_{j 2}\right|,\left|e_{i 2}-e_{j 1}\right|\right)\right)
\end{aligned}
$$

Thus, taking a ground truth segment and a detected one, it is considered that there exists a correct matching between them if the corresponding value of $h d$ is not 
Table 3 Number of detected segments $\left(N S_{d}\right)$ and percentage of connected segments (CS) for the ten test images

\begin{tabular}{|c|c|c|c|c|c|c|}
\hline & \multicolumn{2}{|l|}{ PPHT } & \multicolumn{2}{|l|}{ LSD } & \multicolumn{2}{|l|}{ HT3D } \\
\hline & $N S_{d}$ & CS(\%) & $N S_{d}$ & CS(\%) & $N S_{d}$ & CS(\%) \\
\hline 1 & 352 & 0 & 329 & 21 & 263 & 67 \\
\hline 2 & 434 & 0 & 515 & 13 & 335 & 74 \\
\hline 3 & 1847 & 0 & 751 & 17 & 912 & 69 \\
\hline 4 & 2376 & 0 & 1274 & 15 & 1213 & 77 \\
\hline 5 & 1655 & 0 & 838 & 13 & 907 & 63 \\
\hline 6 & 2768 & 0 & 1034 & 20 & 1321 & 62 \\
\hline 7 & 1079 & 0 & 1058 & 14 & 924 & 76 \\
\hline 8 & 2622 & 0 & 1112 & 14 & 1269 & 62 \\
\hline 9 & 1295 & 0 & 839 & 15 & 738 & 71 \\
\hline 10 & 2194 & 0 & 1301 & 28 & 1355 & 77 \\
\hline
\end{tabular}

The percentage of connected segments is indicative of the ability of a segment detector to provide chains of segments corresponding to the polygonal image contours. According to this measure, the proposed method (HT3D) produces clearly better results than the other two approaches

greater than a maximum distance $d S$. Using this criterion, Table 4 shows the number of correct matchings $\left(N S_{c}\right)$ of the three methods from the total number of ground truth segments $\left(N S_{g}\right)$ considering values of 4,3 , and 2 for $d S$. From the results obtained, it can be seen that PPHT detects a lower number of correct matchings than the other two approaches, even though the total number of detected segments is considerably greater in many cases. LSD clearly improves on the results of PPHT, but the hit rates are generally lower than those provided by HT3D. The difference in performance between HT3D and the other two segment detectors becomes more evident as

Table 4 Comparison of detected segments with the ground truth for the ten test images

\begin{tabular}{|c|c|c|c|c|c|c|c|c|c|c|c|}
\hline & & & $N S_{C}$ & & & & & & & & \\
\hline & & & $\overline{\mathrm{PPH}}$ & & & LSD & & & HT3 & & \\
\hline & $N S_{g}$ & $d S$ & 4 & 3 & 2 & 4 & 3 & 2 & 4 & 3 & 2 \\
\hline 1 & 42 & & 5 & 4 & 3 & 18 & 12 & 4 & 24 & 21 & 9 \\
\hline 2 & 120 & & 21 & 17 & 11 & 64 & 47 & 17 & 75 & 61 & 45 \\
\hline 3 & 111 & & 23 & 13 & 5 & 59 & 43 & 19 & 59 & 47 & 32 \\
\hline 4 & 628 & & 92 & 56 & 20 & 272 & 174 & 61 & 291 & 236 & 124 \\
\hline 5 & 306 & & 58 & 34 & 17 & 120 & 65 & 23 & 124 & 82 & 37 \\
\hline 6 & 434 & & 57 & 34 & 12 & 174 & 112 & 32 & 194 & 158 & 89 \\
\hline 7 & 373 & & 54 & 27 & 9 & 154 & 101 & 46 & 155 & 121 & 73 \\
\hline 8 & 350 & & 70 & 44 & 13 & 159 & 105 & 42 & 155 & 113 & 61 \\
\hline 9 & 139 & & 14 & 11 & 5 & 45 & 30 & 18 & 60 & 50 & 29 \\
\hline 10 & 209 & & 70 & 54 & 36 & 136 & 112 & 45 & 159 & 145 & 92 \\
\hline
\end{tabular}

The number of correct matchings of each method (NSC) is shown considering different values of the distance between ground truth and detected segments (dS). The total number of ground truth segments $\left(\mathrm{NS}_{g}\right)$ is shown in the first column $d S$ decreases. This can be observed, not only in the ten test images but also in the whole dataset, through the results depicted in Figs. 23, 24, 25 and 26. These figures show the ratios $N S_{c} / N S_{d}$ and $N S_{c} / N S_{g}{ }^{4}$ for each image of the dataset considering values of 3 and 2 for $d S$. Regarding the ratio between the number of correct matchings and the total number of detected segments $\left(N S_{c} / N S_{d}\right)$, HT3D gives much greater values than PPHT in almost $100 \%$ of the tests for the different values of $d S$. The main reason is that PPHT detects a high rate of false positives, which considerably diminishes this ratio. They can be reduced by increasing the minimum number of line votes, but at the expense of proportionally reducing the number of correct matchings. In HT3D, false positives are avoided by requiring that Hough cells in contiguous positions to the ones defining a segment do not also define full segments on both sides. This solves to a great extent the problem of detecting false segments, although this means that parallel segments must be situated some distance away from each other in order to be correctly detected.

In relation to the comparison between HT3D and LSD, although the differences are less notable than in the comparison with PPHT, better results are obtained in most cases when using HT3D. Differences between the results of both methods become more obvious for $d S=2$. The principal cause is that segments detected by LSD repeatedly break off before reaching their actual endpoints. In addition, when segments are crossed by other segments, they break into shorter segments, which affects not only the number of correct segments but also the total number of detected segments. Both problems also occur in PPHT (see Fig. 22).

With respect to the ratio $N S_{c} / N S_{g}$ (hit rate), the results obtained lead to similar conclusions. Thus, the hit rate of HT3D remains above the one provided by PPHT in the large majority of tests. In addition, the hit rate of HT3D is higher than the hit rate of LSD in $71 \%$ of the test images with $d S=3$ and above it in $87 \%$ taking $d S=2$. The average difference between the hit rates of both methods is 3.5 and 6.5 percentage points, assuming, respectively, $d S=3$ and $d S=2$. This denotes greater accuracy of the proposed segment detector compared to the other two approaches.

Our rectangle detection method has also been tested using a set of aerial images [41]. Figures 27, 28 and 29 show some results. The method has been tested using a value of 3 for $\Delta d$ and $\Delta p$ and the corresponding value of $\Delta \theta$ obtained by expression 24 . The rectangle strength threshold $\tau_{r}$ has been set to 0.75 . For each image in Fig. 27, the Hough space is formed using the corresponding edge image in Fig. 28. Detected rectangles are depicted in Fig. 29. As it can be observed, despite the complexity of some edge images, the varied size of the constructions, and an imperfect or incomplete border representation of 


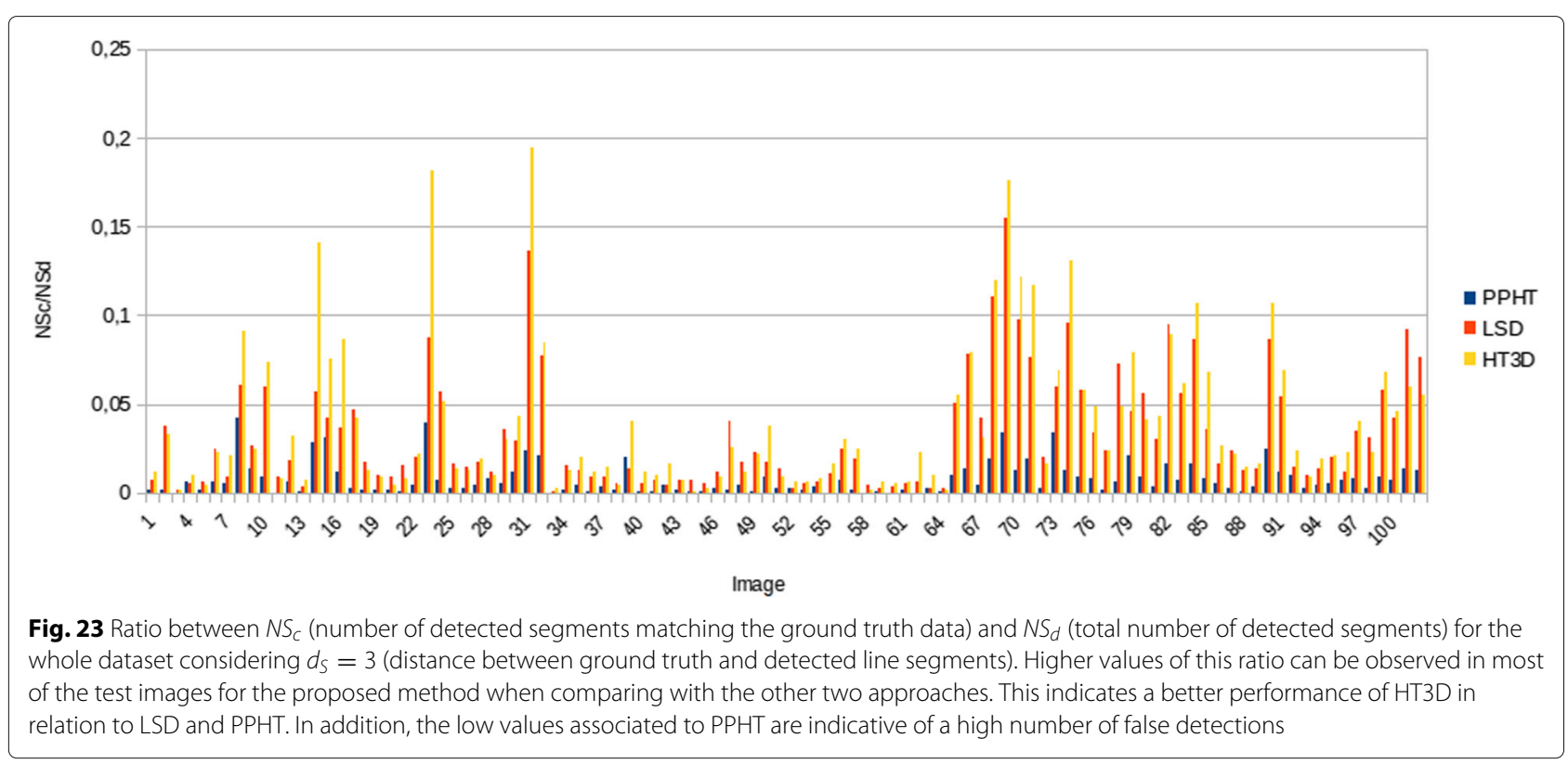

some structures, the proposed method correctly detects most of the rectangular contours. There are some false detections caused by two different factors. False positives corresponding to small areas are due to the fact that these kinds of regions are less affected by edge gaps than bigger ones and so they should be confirmed with higher values of $\tau_{r}$. In this sense, instead of using a constant threshold, a variable threshold obtained as a function of the region size should be applied to improve the results. The other group of false detections corresponds to areas close to actual rectangular regions that share several corners with them. For better performance, those rectangles sharing more than one corner and presenting a similar orientation must be separately analyzed to determine which of them are finally considered. Both improvements, however, are beyond the scope of this paper since they are specific to rectangle detection.

\section{Conclusions}

This paper presents an extension of the Hough Transform, called HT3D, for the combined detection of corners, segments, and polylines. This new variant of HT uses a 3D parameter space that facilitates the detection of segments instead of lines. It has been shown how this representation also encloses canonical configurations of corners and non-intersection endpoints, making it a powerful tool, not only for the detection of line segments but also for the extraction of such kinds of points.

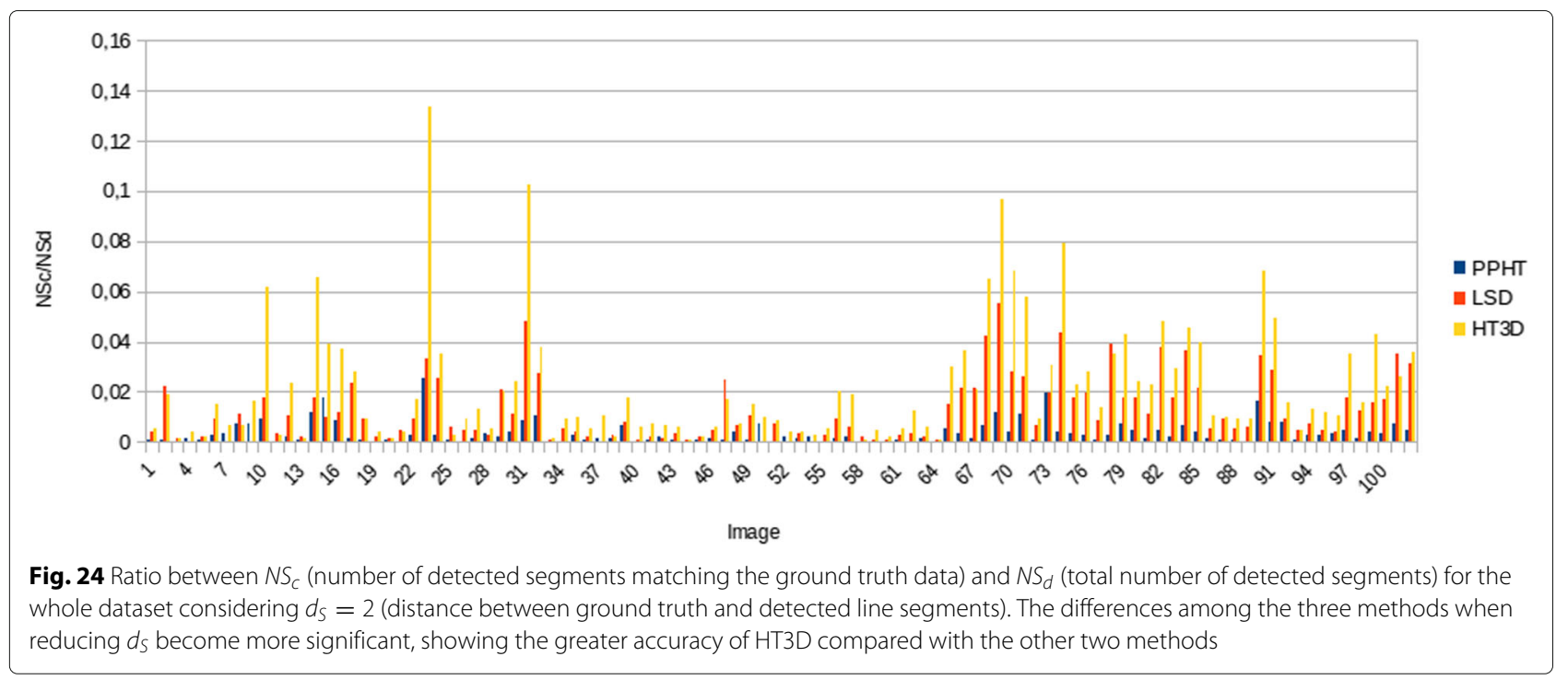




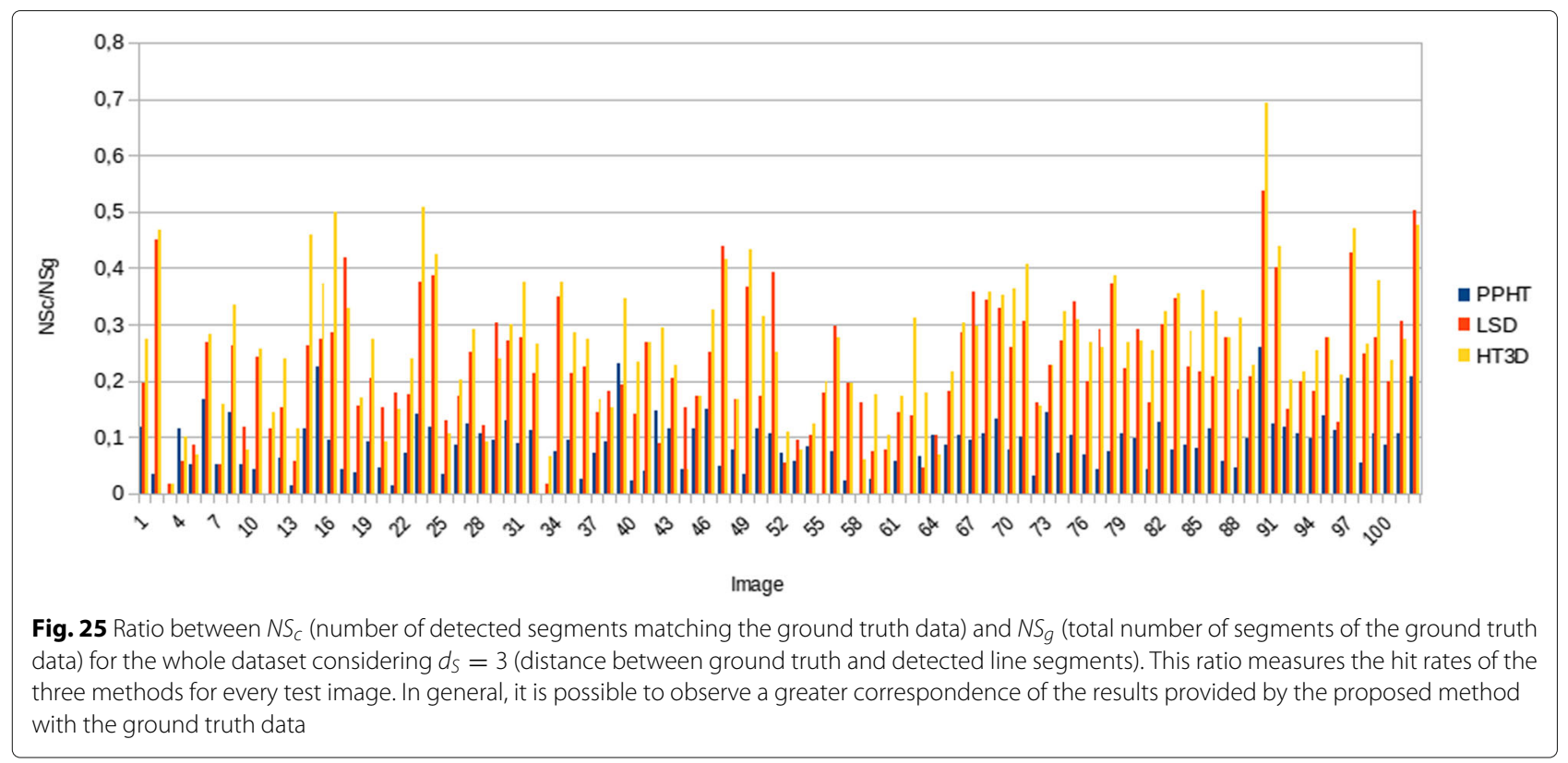

One of the main novelties of our proposal is that line segments are not directly searched for in the image. Instead, they are verified from their endpoints using a segment measure obtained from the segment representation provided by the Hough space. This makes line segment detection robust to line gaps, edge deviations, and crossing lines. In addition, this segment detection strategy improves the accuracy of the results in relation to other approaches. Thus, methods based on the analysis of edge or gradient information in the image space miss the actual boundaries of line segments, since gradient at those points presents a high uncertainty [28]. Instead of building chains of aligned edge pixels, the presented approach confirms the existence of line segments between couples of previously detected endpoints. Experiments have shown how this inverse strategy provides more accurate results than the segment generation approach. The main drawback of using a segment measure is that it could produce high responses for non-real line segments in noisy or complex images. False positives are mainly false segments

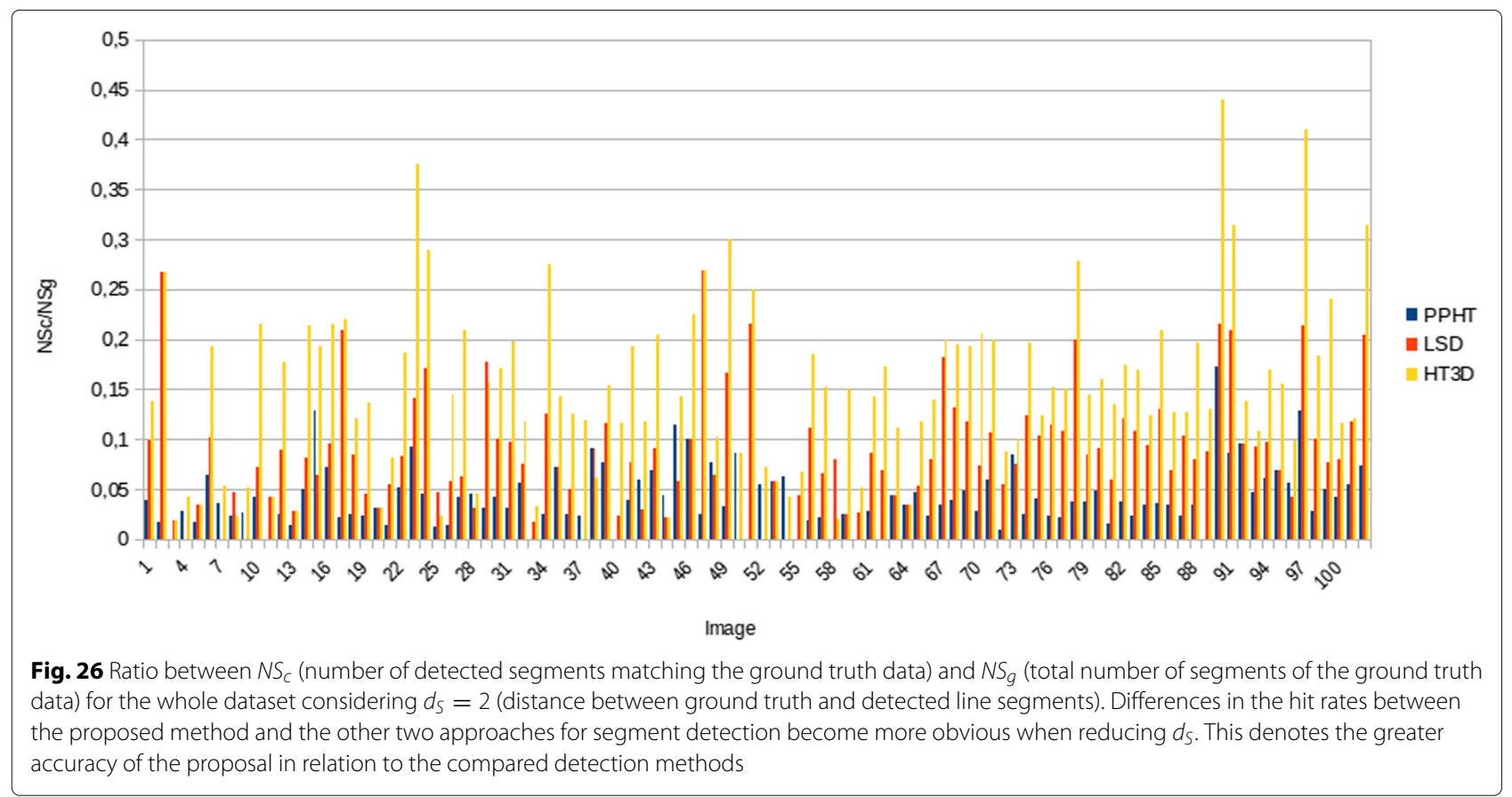



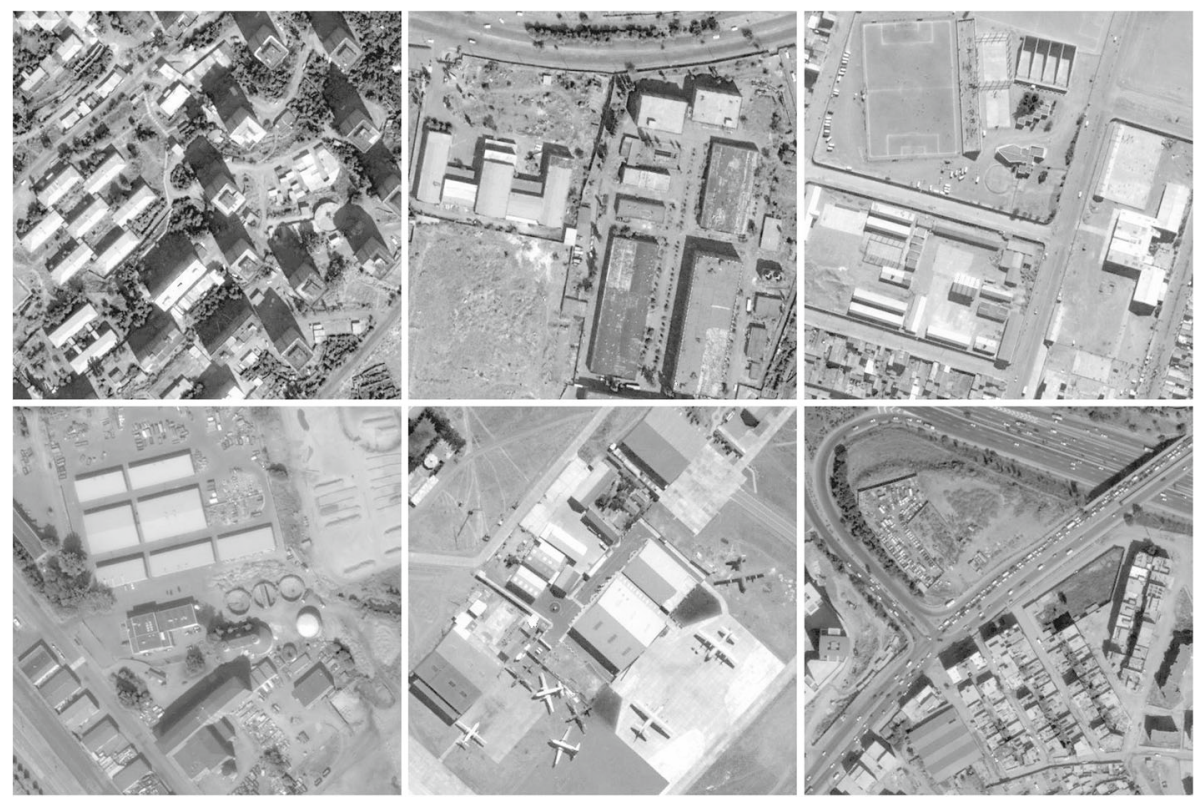

Fig. $\mathbf{2 7}$ Set of images used for testing the proposed rectangle detection method

crossing several real segments. Thus, false detections can be controlled by requiring that the Hough cells situated in contiguous positions to the pair of cells defining a line segment do not produce high values for the segment measure. Depending on the resolution of the Hough space, this additional criterion might discard true positive detections corresponding to close parallel line segments. Nevertheless, it significantly reduces the number of false positives, producing reliable results.
Regarding corner detection, our proposal provides an alternative to intensity-based detectors. These methods present some limitations in the detection of corners of obtuse angles, since in the local environment of those points significant intensity changes are only perceived in one direction [42]. In our approach, corners are considered as image points intersecting two line segments at a given angle range. This corner definition is consistent with certain cell patterns in the Hough space.

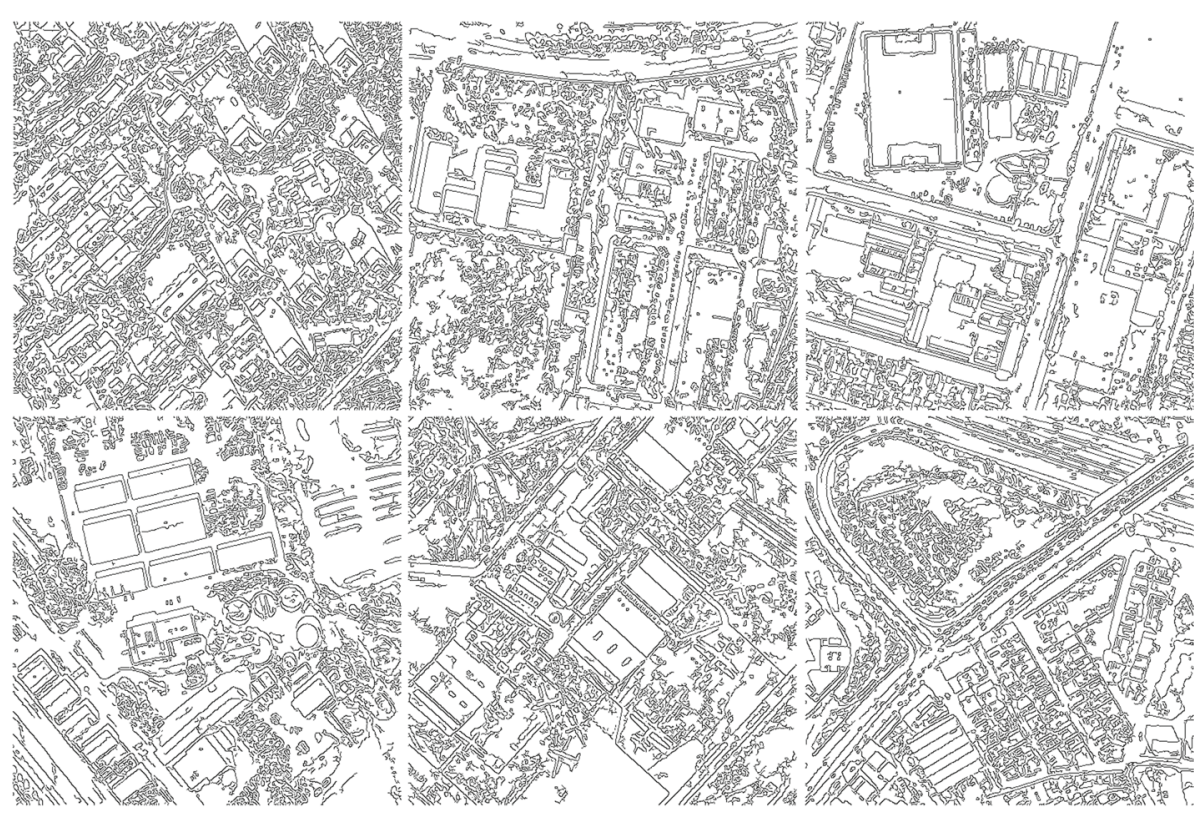

Fig. 28 Edge representation of the images in Fig. 27 

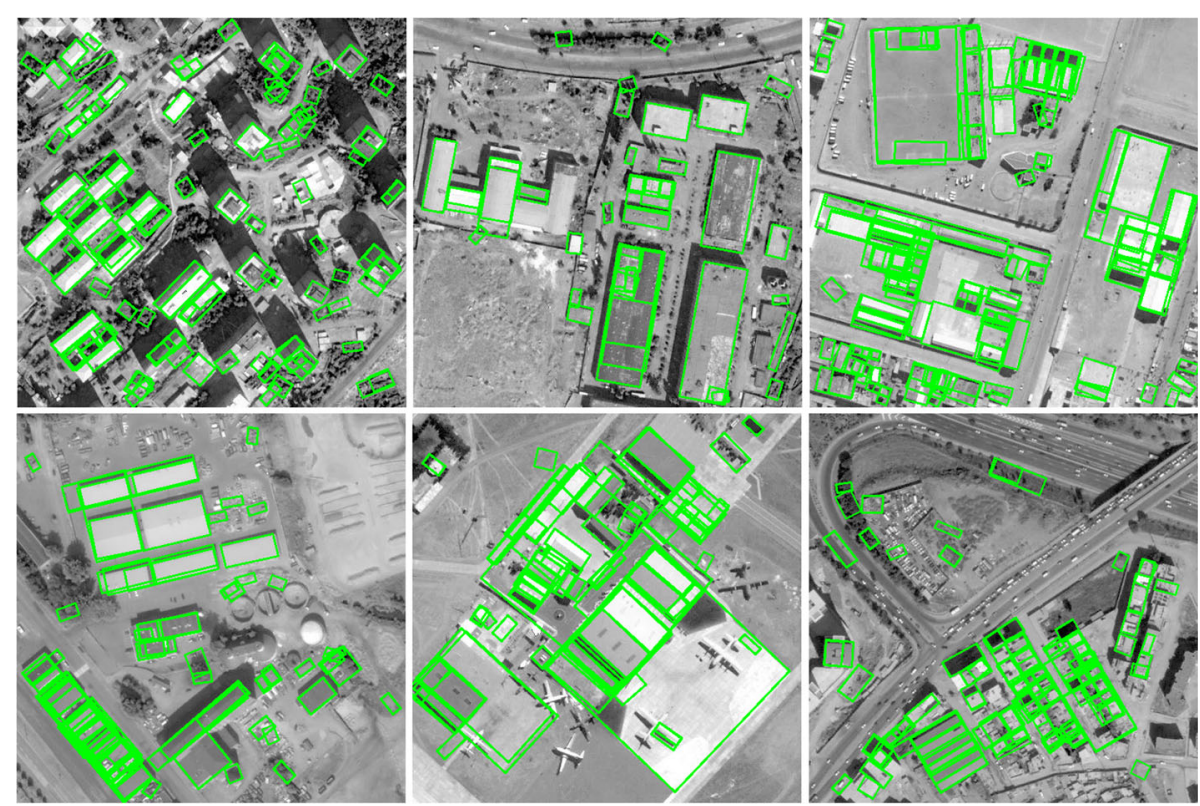

Fig. 29 Detected rectangles in the Fig. 27 images

Thus, applying a pattern matching process, corners are detected in the Hough space and then located in the image space. If a corner is identified in the Hough space, it is accepted as a valid detection and the intensity image is only used to find the most likely pixel position associated to the corresponding corner position in the Hough space. This coarse-to-fine method avoids applying any threshold related to changes of intensity in the corner local environment, which allows the identification of corner points that other methods miss. The same proposed strategy for corner detection is also used for detecting other kinds of distinctive points corresponding to segment endpoints that do not intersect other line segments. This set of points, called non-intersection endpoints, is key for obtaining reliable results in the detection of segments, since segment endpoints do not always correspond to corners. The experimental evaluation presented in this paper shows good detection rates of both types of points when comparing with the ground truth. Thus, our proposal matches up to $20 \%$ more ground truth points than the compared methods.

Besides the accuracy benefits, the combined detection of corners and line segments produces the direct identification of polygonal structures in the image, which has additional applications. Moreover, we have shown how the segment measure can be extended to detect predefined polygonal shapes, which are important image features in a variety of problems, such as those related to the identification of man-made structures in aerial images.

The whole detection method entails five processing stages on a 3D memory structure, so it presents a higher computational complexity than methods devoted to the detection of individual features. Nevertheless, all the stages present a characteristic structure that makes them inherently parallelizable. In this regard, some initial results of a parallel implementation of the method have been obtained, showing a significant time reduction. We are working on this parallel implementation and developing new optimizations to reduce execution times even further.

\section{Endnotes}

${ }^{1}$ Otherwise, the same reasoning can be done using the expressions of $d_{1}$ and $d_{2}$ from $\left(d_{1}^{\prime}, p_{1}^{\prime}\right)$ and $\left(d_{2}^{\prime}, p_{2}^{\prime}\right)$ instead of Eq. 10.

${ }^{2}$ It must be noted that this function is only computed for those pixels detected as potential corners/endpoints in the Hough space and not for the whole image.

${ }^{3}$ These results have been obtained on images of size $640 \times 480$ with around $15 \%$ edge points using an Intel i7 $2.67 \mathrm{GHz}$ processor for the sequential version and a NVIDIA GTX 1060 GPU for the parallel implementation.

${ }^{4}$ Both ratios remain moderate in all the methods for two main reasons. The first one is that the ground truth data only include the set of line segments that conform to the 3D orthogonal frame of the environment. In addition, some marked segments do not have enough intensity to be detected as image edges or they even appear partially occluded in the gray image. 


\section{Acknowledgements}

This work has partly been supported by grants TIN2015-65686-C5-5-R and PHBP14/00083, from the Spanish Government.

\section{Authors' contributions}

PBB wrote the main part of this manuscript. LJM modified the content of the manuscript. PB participated in the discussion. All authors read and approved the final manuscript.

\section{Competing interests}

The authors declare that they have no competing interests.

\section{Publisher's Note}

Springer Nature remains neutral with regard to jurisdictional claims in published maps and institutional affiliations.

\section{Received: 2 August 2016 Accepted: 12 April 2017}

Published online: 02 May 2017

\section{References}

1. A De la Escalera, JM Armingol, Automatic chessboard detection for intrinsic and extrinsic camera parameter calibration. Sensors. 10(3), 2027-2044 (2010)

2. L Forlenza, P Carton, D Accardo, G Fasano, A Moccia, Real time corner detection for miniaturized electro-optical sensors onboard small unmanned aerial systems. Sensors. 12(1), 863-877 (2012)

3. R Chandratre, VA Chakkarwar, Article: Image stitching using harris and ransac. Int. J. Comput. Appl. 89(15), 14-19 (2014)

4. A Dick, P Torr, R Cipolla, in Proceedings of the British Machine Vision Conference. Automatic $3 \mathrm{~d}$ modelling of architecture (BMVA Press, UK, 2000), pp. 39-13910

5. P Kahn, LJ Kitchen, EM Riseman, A fast line finder for vision-guided robot navigation. IEEE Trans. Pattern Anal. Mach. Intell. 12(11), 1098-1102 (1990)

6. CX Ji, ZP Zhang, Stereo match based on linear feature. ICPR. 88, 875-878 (1988)

7. P Fränti, E Ageenko, H Kälviäinen, S Kukkonen, in Proc. Fourth Joint Conference on Information Sciences JCIS'98. Compression of Line Drawing Images Using Hough Transform for Exploiting Global Dependencies (Association for Intelligent Machinery, Research Triangle Park, 1998), pp. 433-436

8. H Moon, R Chellappa, A Rosenfeld, Performance analysis of a simple vehicle detection algorithm. Image Vis. Comput. 20(1), 1-13 (2002)

9. S Noronha, R Nevatia, Detection and modeling of buildings from multiple aerial images. IEEE Trans. Pattern Anal. Mach. Intell. 23(5), 501-518 (2001)

10. Y Zhu, B Carragher, F Mouche, CS Potter, Automatic particle detection through efficient Hough transforms. IEEE Trans. Med. Imaging. 22(9), 1053-1062 (2003)

11. PVC Hough, Method and means for recognizing complex patterns. Google Patents. Patent 3, US 069,654 (1962). http://www.google.com/ patents/US3069654. Accessed Apr 2017

12. P Mukhopadhyay, BB Chaudhuri, A survey of Hough Transform. Pattern Recognit. 48(3), 993-1010 (2014)

13. RO Duda, PE Hart, Use of the Hough transformation to detect lines and curves in pictures. Commun. ACM. 15(1), 11-15 (1972)

14. Y Ching, Detecting line segments in an image-a new implementation for Hough Transform. Pattern Recognit. Lett. 22(3/4), 421-429 (2001)

15. H Duan, X Liu, H Liu, in Pervasive Computing and Applications, 2007. ICPCA 2007. 2nd International Conference On. A nonuniform quantization of hough space for the detection of straight line segments (IEEE, Birmingham, 2007), pp. 149-153

16. LAF Fernandes, MM Oliveira, Real-time line detection through an improved Hough transform voting scheme. Pattern Recognit. 41(1), 299-314 (2008)

17. J Song, MR Lyu, A hough transform based line recognition method utilizing both parameter space and image space. Pattern Recognit. 38(4), 539-552 (2005)

18. G Gerig, in Proc. of First International Conference on Computer Vision. Linking image-space and accumulator-space: a new approach for object recognition (Computer Society Press of the IEEE, London, 1987), pp. 112-117
19. J Matas, C Galambos, J Kittler, Robust detection of lines using the progressive probabilistic hough transform. Comput. Vis. Image Underst. 78(1), 119-137 (2000)

20. TT Nguyen, XD Pham, J Jeon, in Proc. IEEE Int. Conf. Industrial Technology. An improvement of the standard hough transform to detect line segments (IEEE, Chengdu, 2008), pp. 1-6

21. J Cha, RH Cofer, SP Kozaitis, Extended Hough transform for linear feature detection. Pattern Recognit. 39(6), 1034-1043 (2006)

22. S Du, BJ van Wyk, C Tu, X Zhang, An improved Hough transform neighborhood map for straight line segments. Trans. Img. Proc. 19(3), 573-585 (2010)

23. S Du, C Tu, BJ van Wyk, EO Ochola, Z Chen, Measuring straight line segments using ht butterflies. PLOS ONE. 7(3), 1-13 (2012)

24. Z Xu, B-S Shin, in Image and Video Technology. Lecture Notes in Computer Science. Line segment detection with Hough transform based on minimum entropy, vol. 8333 (Springer, Berlin Heidelberg, 2014), pp. 254-264

25. ER Davies, Application of the generalised hough transform to corner detection. IEE Proc. E (Comput. Digit. Tech.) 135, 49-545 (1988)

26. DH Ballard, Readings in computer vision: issues, problems, principles, and paradigms, Chap. Generalizing the Hough Transform to Detect Arbitrary Shapes. (Morgan Kaufmann Publisher Inc., San Francisco, 1987), pp. 714-725

27. WA Barrett, KD Petersen, in Computer Vision and Pattern Recognition, 2001. CVPR 2001. Proceedings of the 2001 IEEE Computer Society Conference On, vol. 2. Houghing the hough: peak collection for detection of corners, junctions and line intersections (IEEE, Kauai, 2001), pp. 302-3092

28. F Shen, H Wang, Corner detection based on modified hough transform. Pattern Recognit. Lett. 23(8), 1039-1049 (2002)

29. J Shi, C Tomasi, in 1994 IEEE Conference on Computer Vision and Pattern Recognition (CVPR'94). Good features to track (Cornell University, Ithaca, 1994), pp. 593-600

30. Buluç, H Meyerhenke, I Safro, P Sanders, C Schulz, Recent advances in graph partitioning. CoRR. abs/1311.3144, 1-37 (2013)

31. MA Gutierrez, P Bachiller, L Manso, P Bustos, Nú, P, ñez, in Proceedings of the $5^{\text {th }}$ European Conference on Mobile Robots, ECMR 2011, September 7-9, 2011, Örebro, Sweden. An incremental hybrid approach to indoor modeling (Learning Systems Lab, AASS, Örebro University, Örebro, 2011), pp. 219-226

32. P Bachiller, $\mathrm{P}$ Bustos, $L \mathrm{~L}$ Manso, in Advances in Stereo Vision, ed. by JRA Torreao. Attentional behaviors for environment modeling by a mobile robot (InTech, Croatia, 2011), pp. 17-40

33. V Shapiro, Accuracy of the straight line hough transform: The non-voting approach. Comp. Vision Image Underst. 103(1), 1-21 (2006)

34. M Zhang, in In Proc. of IEEE (ICPR'96). On the discretization of parameter domain in Hough transformation (IEEE Computer Society, Los Alamitos, 1996), pp. 527-531

35. P Denis, JH Elder, FJ Estrada, in ECCV (2). Lecture Notes in Computer Science, ed. by DA Forsyth, PHS Torr, and A Zisserman. Efficient edge-based methods for estimating manhattan frames in urban imagery, vol. 5303 (Springer, Berlin Heidelberg, 2008), pp. 197-210

36. JM Coughlan, AL Yuille, Manhattan world: orientation and outlier detection by bayesian inference. Neural Comput. 15, 1063-1088 (2003)

37. C Harris, M Stephens, in Proc. of Fourth Alvey Vision Conference. A combined corner and edge detector (Organising Committee AVC 88, Manchester, 1988), pp. 147-151

38. E Rosten, T Drummond, in Proceedings of the 9th European Conference on Computer Vision - Volume Part I. ECCV'06. Machine learning for high-speed corner detection (Springer-Verlag, Berlin, 2006), pp. 430-443

39. R Grompone von Gioi, J Jakubowicz, J Morel, G Randall, Lsd: a line segment detector. Image Processing On Line. 2, 35-55 (2012)

40. J Henrikson, Completeness and total boundedness of the Hausdorff metric. MIT Undergrad. J. Math. 1, 69-80 (1999)

41. J Yuan, SS Gleason, AM Cheriyadat, Systematic benchmarking of aerial image segmentation. IEEE Geosci. Remote Sensing Lett. 10(6), 1527-1531 (2013)

42. S Kim, C Park, Y Choi, S Kwon, IS Kweon, Feature point detection by combining advantages of intensity-based approach and edge-based approach. Int. J. Comput. Electr. Autom. Control Inform. Eng. 6(8), 1055-1060 (2012) 